\title{
EULER CHARACTERISTICS OF THE REAL POINTS OF CERTAIN VARIETIES OF ALGEBRAIC TORI
}

\author{
G.I. LEHRER AND J. VAN HAMEL
}

\begin{abstract}
Let $G$ be a complex connected reductive group which is defined over $\mathbb{R}$, let $\mathfrak{G}$ be its Lie algebra, and $\mathcal{T}$ the variety of maximal tori of $G$. For $\xi \in \mathfrak{G}(\mathbb{R})$, let $\mathcal{T}_{\xi}$ be the variety of tori in $\mathcal{T}$ whose Lie algebra is orthogonal to $\xi$ with respect to the Killing form. We show, using the Fourier-Sato transform of conical sheaves on real vector bundles, that the "weighted Euler characteristic" (see below) of $\mathcal{T}_{\xi}(\mathbb{R})$ is zero unless $\xi$ is nilpotent, in which case it equals $(-1)^{\frac{\operatorname{dim} \mathcal{T}}{2}}$. This is a real analogue of a result over finite fields, which is connected with the Steinberg representation of a reductive group.
\end{abstract}

\section{INTRODUCTION AND NOTATION}

Let $X$ be a complex algebraic variety which is defined over $\mathbb{R}$ (see [BT, Bo, Spr] for background concerning this notion). The Galois group $\Gamma:=\operatorname{Gal}(\mathbb{C} / \mathbb{R})=\langle\sigma\rangle$ acts on $X$ continuously (in fact anti-holomorphically), and we speak of the fixed points $X^{\sigma}:=X(\mathbb{R})$ as the variety of real points of $X$. This is known [W] to have a finite number of path connected components, each of which is an analytic variety of dimension equal to $\operatorname{dim} X$. In this work, we present two results concerning the "weighted Euler characteristic" of $X(\mathbb{R})$, when $X$ is a certain variety associated with a complex reductive group $G$ which is defined over $\mathbb{R}$. Specifically, our varieties $X$ will be closed subvarieties of the variety of maximal tori of $G$. Here "weighted Euler characteristic" means the sum of the Euler characteristics of the components of $X(\mathbb{R})$, each one weighted by a sign which will be defined shortly.

Our results here are analogues of those of [Le2], where the varieties considered were over fields of positive characteristic, and where the Frobenius endomorphism played the role of complex conjugation. The weighted Euler characteristics which we compute are real analogues of the character formula for the Steinberg function on a finite Lie algebra and its Fourier transform (see [Le2, Le1]). Our main results below may be regarded as a realisation of the remarks [Le2, p. 49].

The proof of these results depends on the construction of two complexes of equivariant sheaves on the Lie algebra of $G$, one of which is known to be perverse, which have equal "characteristic functions" (see $\$ 7.2$ below). We then employ an equivariant version of the Fourier-Sato transform functor on the derived category of the category of equivariant conical sheaves on a real vector bundle, as expounded by Kashiwara 
and Schapira $[\mathrm{KS}]$. After showing that the Fourier transform functor respects characteristic functions, we apply the Fourier transform to our complexes, and our results emerge when the respective characteristic functions are identified.

It is an interesting question whether the complexes mentioned are actually equal in the derived category of equivariant sheaves.

1.1. Description of the main results. Let $X$ be a complex variety with a real structure as above. Let $\mathbb{F}$ be a field of characteristic zero, and suppose $\mathcal{L}$ is a local system (i.e. locally constant sheaf) of $\mathbb{F}$-vector spaces on $X$, which is $\Gamma$-equivariant in the sense that we have an isomorphism of sheaves $\alpha_{\sigma}: \mathcal{L} \stackrel{\sim}{\longrightarrow} \sigma^{*} \mathcal{L}$. Then $\alpha_{\sigma}$ induces endomorphisms which we refer to as $\sigma$, of the cohomology of $\mathcal{L}$ with compact supports, $H_{c}^{i}(X, \mathcal{L})$, and we define

$$
\Lambda_{c}(\sigma, X, \mathcal{L})=\sum_{i}(-1)^{i} \operatorname{Trace}\left(\sigma, H_{c}^{i}(X, \mathcal{L})\right)
$$

Our main results give explicit values of $\Lambda_{c}(\sigma, X, \mathcal{L})$ for particular pairs $X, \mathcal{L}$. In $\S 4$ we explain how $\Lambda_{c}(\sigma, X, \mathcal{L})$ may be interpreted as a weighted Euler characteristic (see Theorem 4.5), as follows. The subspace of real points of $X$ is a finite disjoint union $X^{\sigma}=\amalg_{C} C$ of connected components, and for $x \in C$, the value of $\operatorname{Trace}\left(\sigma, \mathcal{L}_{x}\right)$ depends only on $C$. Writing $\operatorname{Trace}\left(\sigma,\left.\mathcal{L}\right|_{C}\right)$ for this common value, we have

$$
\Lambda_{c}(\sigma, X, \mathcal{L})=\sum_{C} \operatorname{Trace}\left(\sigma,\left.\mathcal{L}\right|_{C}\right) \chi_{c}(C),
$$

where $\chi_{c}$ denotes the Euler characteristic with compact supports.

Let $G$ be a connected reductive linear algebraic group over $\mathbb{C}$, which is defined over $\mathbb{R}$. We write $\mathcal{B}$ for the variety of Borel subgroups of $G, \mathcal{T}$ for the variety of maximal tori of $G$ and $\mathcal{P}$ for the variety of "Killing pairs" (cf. [SGA3, Exposé XXII]) $\{(B, T) \mid B \in \mathcal{B}, T \in \mathcal{T}, B \supseteq T\}$. The varieties $\mathcal{B}, \mathcal{T}$ and $\mathcal{P}$ all have real structures, and the second projection $p: \mathcal{P} \longrightarrow \mathcal{T}$ is a Galois covering whose Galois group is the "absolute Weyl group" $W$ of $G$. If $\mathbb{F}$ denotes the constant sheaf on $\mathcal{P}$, the pushdown $p_{!} \mathbb{F}$ is the local system on $\mathcal{T}$ which corresponds to the regular representation of $W$. Assuming $\mathbb{F}$ to be algebraically closed, we have a decomposition

$$
p_{!} \mathbb{F}=\oplus_{E \in \hat{W}} E \otimes \mathcal{S}_{E},
$$

where $\mathcal{S}_{E}$ is the irreducible local system on $\mathcal{T}$ which corresponds to the irreducible representation $E$ of $W$. The local system $\mathcal{S}_{E}$ is $\Gamma$-equivariant precisely when the representation ${ }^{\sigma} E$ of $W$ is equivalent to $E$ (in the obvious notation).

The automorphism which $\sigma$ induces on $W$ is realised as a linear conjugation in the representation of $W$ as a reflection group. Hence reflections are taken to reflections, and it follows that the invariance condition above is satisfied when $E=\epsilon$, the sign (or alternating) representation of $W$. This leads to the

Definition 1.4. The $\Gamma$-equivariant local system $\mathcal{S}_{\epsilon}$ on $\mathcal{T}$ is the local system $\mathcal{S}_{E}$ of (1.3), with $E=\epsilon$.

Now let $\mathfrak{G}$ be the Lie algebra of $G$. Clearly $\mathfrak{G}$ is also defined over $\mathbb{R}$ and inherits a $\sigma$-action. Moreover $\mathfrak{G}$ has an $\operatorname{Ad}(G)$ invariant non-degenerate form which we may 
assume is defined over $\mathbb{R}$. When $\mathfrak{G}$ is semisimple, this may be taken to be the Killing form. We write this form $\langle\rangle:, \mathfrak{G} \times \mathfrak{G} \longrightarrow \mathbb{C}$, and note that $\langle\mathfrak{G}(\mathbb{R}), \mathfrak{G}(\mathbb{R})\rangle \subset \mathbb{R}$.

Definition 1.5. Let $\xi \in \mathfrak{G}(\mathbb{R})$ and define $\mathbb{R}$-varieties $\mathcal{T}^{\xi}$ and $\mathcal{T}_{\xi}$ as follows.

$$
\begin{gathered}
\mathcal{T}^{\xi}=\{T \in \mathcal{T} \mid \operatorname{Lie} T \ni \xi\} \\
\mathcal{T}_{\xi}=\{T \in \mathcal{T} \mid\langle\operatorname{Lie} T, \xi\rangle=0\} .
\end{gathered}
$$

Observe that $T^{\xi}$ is empty if $\xi$ is not semisimple.

Our main results are the next two theorems.

Theorem 1.8. With notation as indicated above, we have for any semisimple element $\xi \in \mathfrak{G}(\mathbb{R})$,

$$
\sum_{\substack{C \subset \mathcal{T}^{\xi}(\mathbb{R}) \\ \text { connected } \\ \text { component }}} \chi_{c}(C) \cdot \epsilon(C)=\epsilon_{0}(G) \Lambda_{c}\left(\sigma, \mathcal{T}^{\xi}, \mathcal{S}_{\epsilon}\right)=\epsilon_{0}\left(Z_{G}(\xi)^{0}\right) \epsilon\left(v_{\xi}\right)(-1)^{N(\xi)}= \pm 1,
$$

where $\epsilon(C)$ is the common value of the sign (see (2.3)) of the maximal tori in the connected component $C, \epsilon_{0}(H)$ is the index (2.7) of any connected reductive group $H$ defined over $\mathbb{R}, v_{\xi}$ is the type (2.6) of a maximally split torus of $Z_{G}(\xi)^{0}$, and $N(\xi)$ is the number of positive roots of $Z_{G}(\xi)^{0}$.

Theorem 1.10. Maintaining the above notation, we have for any element $\xi \in \mathfrak{G}(\mathbb{R})$,

$$
\sum_{\substack{C \subset \mathcal{T}_{\xi}(\mathbb{R}) \\
\text { connected } \\
\text { component }}} \chi_{c}(C) \cdot \epsilon(C)=\epsilon_{0}(G) \Lambda_{c}\left(\sigma, \mathcal{T}_{\xi}, \mathcal{S}_{\epsilon}\right)=\left\{\begin{array}{l}
(-1)^{N} \epsilon_{0}(G) \text { if } \xi \text { is nilpotent } \\
0 \text { otherwise }
\end{array}\right.
$$

where $N$ is the number of positive roots of $G$.

1.2. Outline of the basic strategy. For any $\sigma$-stable maximal torus $T$ of $G$, the covering $G / T \rightarrow \mathcal{T}$ is a Galois twist (see (3.20), (3.18) in $\S 3$ ) of $p: \mathcal{P} \rightarrow \mathcal{T}$. Among the $G(\mathbb{R})$-conjugacy classes of maximal tori in $\mathcal{T}^{\sigma}$, there is a distinguished one consisting of "maximally split" tori (see Lemma 2.1 below); this enables us to attach a sign $\epsilon(T)$ to any maximal torus $T \in \mathcal{T}(\mathbb{R})$. Sections 2 and 3 are devoted to the study of these signs and twists, and their Galois cohomological classification using $H^{1}(\Gamma, W)$. This leads to the proof in $\S 5$ of Theorem 1.8, which is fairly straightforward, and proceeds essentially by induction on the semisimple rank of $G$.

The proof of Theorem 1.10 involves the Fourier-Sato transform construction mentioned above. Let $E$ be a complex vector bundle defined over $\mathbb{R}$ with its antiholomorphic involution $\sigma$. The category $\mathcal{D}_{c c}^{b}(E ; \sigma, \mathbb{F})$ is the category of complexes of $\sigma$-equivariant $\mathbb{F}$-vector space sheaves on $E$, whose cohomology sheaves are conical (see (6.1.1) below) and constructible with respect to some $\sigma$-equivariant semi-algebraic stratification. Sections 6 and 7 are devoted to an exposition of the basic properties of this category, and of the Fourier-Sato transform functor $\mathcal{F}_{E}: \mathcal{D}_{c c}^{b}(E ; \sigma, \mathbb{F}) \longrightarrow$ $\mathcal{D}_{c c}^{b}(\check{E} ; \sigma, \mathbb{F})$, where $\check{E}$ is the dual of the bundle $E$. This is based largely on [BBD, KS].

If $K^{\bullet}$ is a complex in $\mathcal{D}_{c c}^{b}(E ; \sigma, \mathbb{F})$, we define the orbit characteristic function $\chi_{K} \bullet$ : $E / \Gamma \longrightarrow R(\Gamma)$, where $R(\Gamma)$ is the representation ring of $\Gamma$ (see $(7.13)$ ), and the 
associated characteristic function $\Lambda_{K} \bullet: E(\mathbb{R}) \rightarrow \mathbb{F}$, which is given by taking the trace of $\sigma$ on $\chi_{K} \bullet(\xi)$, for $\xi \in E^{\sigma}=E(\mathbb{R}) \subseteq E / \Gamma$. A key property (see Proposition 7.15) of these functions is that if $K^{\bullet}$ and $M^{\bullet}$ are complexes in $\mathcal{D}_{c c}^{b}(E ; \sigma, \mathbb{F})$ such that $\chi_{K^{\bullet}}=$ $\chi_{N^{\bullet}}$, then $\chi_{\mathcal{F}_{E} K^{\bullet}}=\chi_{\mathcal{F}_{E} N^{\bullet}}$. This is used in the proof of Theorem 1.10 as follows.

First, we define a complex $K^{\bullet} \in \mathcal{D}_{c c}^{b}(\mathfrak{G} ; \sigma, \mathbb{F})$ for which it is easy to see that the characteristic function $\chi_{K} \bullet: \mathfrak{G}(\mathbb{R}) \longrightarrow \mathbb{F}$ coincides with the function $\xi \mapsto \Lambda_{c}\left(\sigma, \mathcal{T}^{\xi}, \mathcal{S}_{\epsilon}\right)$ (for $\xi \in \mathfrak{G}(\mathbb{R})$ ). We then show that there is a shift $M^{\bullet} \in \mathcal{D}_{c c}^{b}(\mathfrak{G})$ of a perverse sheaf in $\mathcal{D}_{c c}^{b}(\mathfrak{G})$, whose orbit characteristic function coincides with that of $K^{\bullet}$. The complex $M^{\bullet}$ arises from Lusztig's construction of the Springer representations of $W$ from the Grothendieck-Springer covering of $\mathfrak{G}$. This is carried out in $\S 8$. Finally we compare the characteristic functions of the Fourier-Sato transforms of the two complexes and use Proposition 7.15 to obtain the formula (1.11) of Theorem 1.10.

1.3. Notation. If $H$ is a subgroup of $G$ and $x \in G$, we denote by ${ }^{x} G$ the conjugate $x G x^{-1}$. If $\alpha$ is an automorphism of $H$, we write $\alpha(h)$, or equally ${ }^{\alpha} h$, for its action, and ${ }^{\alpha} M$ for the twist of an $H$-module $M$ by $\alpha$ (where $h \in H$ acts on $M$ via ${ }^{\alpha} h$ ).

For any complex variety $X$ with a real structure as above, a point $x \in X$ or subvariety $Y \subseteq X$ will be called real if it is $\sigma$-stable.

\section{Maximal tori in algebraic groups Defined over $\mathbb{R}$}

As in $\S 1$, let $G$ be a complex connected reductive linear algebraic group defined over $\mathbb{R}$. Let $T_{0}$ be a real maximal torus of $G$ which contains a maximal $\mathbb{R}$-split torus $S$ of $G$.

Lemma 2.1. The maximal torus $T_{0}$ is unique up to conjugacy under $G(\mathbb{R})$.

Proof. If $T$ is another real maximal torus containing $S$, then $T_{0} / S$ and $T / S$ are anisotropic maximal tori of $Z_{G}(S) / S$, which is again a reductive group defined over $\mathbb{R}$. Since two compact maximal tori of a connected reductive Lie group $H$ are conjugate in $H$, it follows that $T_{0} / S$ and $T / S$ are conjugate by an element of $\left(Z_{G}(S) / S\right)(\mathbb{R})$. Since $S$ is $\mathbb{R}$-split, the Galois cohomology $H^{1}(\mathbb{R}, S)$ is trivial by Hilbert's Theorem 90 (cf. [Se, p. 51]), the map $Z_{G}(S)(\mathbb{R}) \longrightarrow Z_{G}(S) / S(\mathbb{R})$ is surjective, and hence $T$ and $T_{0}$ are conjugate under $G(\mathbb{R})$. Finally, by [Spr, 15.2.6], any two maximal split tori of $G$ are also conjugate under $G(\mathbb{R})$, which proves the assertion.

It follows from Lemma 2.1 that there is a distinguished $G(\mathbb{R})$ conjugacy class of "maximally split" real maximal tori in $G$. Suppose $T_{0}$ is as above, and let $N_{0}:=$ $N_{G}\left(T_{0}\right)$, and $W_{0}:=N_{0} / T_{0}$. These groups all have a $\Gamma$ action. The Weyl group $W_{0}$ has a natural action on $T_{0}$, and hence on the character group $X\left(T_{0}\right)=\operatorname{Hom}\left(T_{0}, \mathbb{C}^{\times}\right)$ and on the co-character group $Y\left(T_{0}\right)=\operatorname{Hom}\left(\mathbb{C}^{\times}, T_{0}\right)$. The involution $\sigma$ also acts on $T_{0}$, and we denote by $\left\langle W_{0}, \sigma\right\rangle$ the group of automorphisms generated by $\sigma$ and the elements of $W_{0}$. If $w \in W_{0}, \sigma(w)$ denotes the image in $W_{0}$ of $w$ under the $\sigma$-action on $W_{0}$, while $\sigma w$ denotes the composite homeomorphism $\sigma \circ w$ of $T_{0}$.

Suppose $T$ is any real maximal torus of $G$. Then $T=g T_{0} g^{-1}$ for some $g \in G$, and since $\sigma T=T, g^{-1} \sigma(g) \in N_{0}$, and using the surjection $N_{0} \longrightarrow W_{0}$, we obtain an element $w \in W_{0}$, which is easily checked to be uniquely determined by $T$ up to $\sigma$-conjugacy, where $w_{1}, w_{2}$ are $\sigma$-conjugate if there exists $w \in W_{0}$ such that $w_{2}=$ 
$w w_{1} \sigma\left(w^{-1}\right)$. If $\epsilon$ is the sign character of $W_{0}$, then since $\sigma$ takes reflections to reflections, we have for $w \in W_{0}, \epsilon(\sigma(w))=\epsilon(w)$, whence

Lemma 2.2. If $w_{1}$ and $w_{2}$ are $\sigma$-conjugate elements of $W_{0}$, then $\epsilon\left(w_{1}\right)=\epsilon\left(w_{2}\right)$.

It follows from Lemma 2.2 and the above discussion, that we may now make the

Definition 2.3. Let $T$ be a maximal torus of $G$ which is defined over $\mathbb{R}$. The sign $\epsilon(T)$ of $T$ is defined as $\epsilon(w)$, where $w \in W_{0}$ is any element of the $\sigma$-conjugacy class of $W_{0}$ corresponding to $T$ as above.

For any group $H$ with a $\sigma$-action, let us write $H_{\sigma}$ for the set $\left\{x \in H \mid \sigma(x)=x^{-1}\right\}$, and denote by $\lambda: H \rightarrow H$ the map $\lambda(x)=x^{-1} \sigma(x)$. Clearly $\lambda(H) \subseteq H_{\sigma}$. Moreover, the set of $\sigma$-conjugacy classes of $H_{\sigma}$ can be indentified with the Galois cohomology group $H^{1}(\Gamma, H)$ (see $\S 3.1$ ). If $T$ is a maximal torus defined over $\mathbb{R}$ as in Definition (2.3), the element $w \in W_{0}$ associated above to $T$ lies in $\left(W_{0}\right)_{\sigma}$, and so the above construction associates with $T$ a well-defined element of $H^{1}\left(\Gamma, W_{0}\right)$. It is easy to see from the definition that this map is constant on $G(\mathbb{R})$-conjugacy classes of tori.

Proposition 2.4. The map $\mathcal{T}(\mathbb{R}) / G(\mathbb{R}) \longrightarrow H^{1}\left(\Gamma, W_{0}\right)$ described above is injective.

Proof. Suppose $g, h \in G$ are such that $\lambda(g)$ and $\lambda(h)$ are in the same $\sigma$-conjugacy class of $W_{0}$. We want to show that ${ }^{g} T_{0}$ and ${ }^{h} T_{0}$ are conjugate under $G(\mathbb{R})$. After modification by some $v \in W_{0}$, we may suppose that $\lambda(g)=\lambda(h) t$, for some element $t \in T_{0}$. Then writing $y=g h^{-1}$, we have $y^{-1} \sigma(y)=\sigma(h) t \sigma(h)^{-1}=t^{\prime} \in(T)_{\sigma}$, where $T=\sigma(h) T_{0} \sigma(h)^{-1}=h T_{0} h^{-1}$. By [She, Cor. 2.3], it follows that $y^{-1} \sigma(y) \in T_{\sigma}^{0}$, where $T_{\sigma}^{0}$ is the connected component of the identity of the real Lie group $T_{\sigma}$. Since $\lambda: T \rightarrow T_{\sigma}^{0}$ is surjective for any complex torus $T$ defined over $\mathbb{R}$, a fact which is easily checked, there is an element $t \in T$ such that $\lambda(y)=\lambda(t)$, whence $\lambda\left(t y^{-1}\right)=1$, i.e. $G^{\sigma} \ni t y^{-1}=r \in G(\mathbb{R})$. But $t=h t_{0} h^{-1}$ for some $t_{0} \in T_{0}$, so that $t y^{-1}=h t_{0} g^{-1}=r$, whence $h t_{0}=r g$. Hence ${ }^{h} T_{0}={ }^{h t_{0}} T_{0}={ }^{r}\left({ }^{g} T_{0}\right)$ as required.

Remark 2.5. Although the map of (2.4) is not generally surjective, it can be shown to be surjective in certain cases, e.g. when $G=\mathrm{GL}_{n}$ (a straightforward calculation) or when $G$ is quasi-split (cf. [Gi], [R]).

Definition 2.6. $\quad$ (i) If $T \in \mathcal{T}(\mathbb{R})$, we shall refer to the image of $T$ under the map in Proposition 2.4 as the type of T. By abuse of notation, we regard the type as an element of $\left(W_{0}\right)_{\sigma} \subset W_{0}$.

(ii) If $\xi$ is a semisimple element of $\mathfrak{G}(\mathbb{R})$, the connected centraliser $Z_{G}(\xi)^{0}$ is a connected reductive group defined over $\mathbb{R}$. It therefore has a maximally split maximal torus $T_{\xi}$, unique up to conjugacy by $Z_{G}(\xi)^{0}(\mathbb{R})$. The type of $T_{\xi}$ as a maximal torus of $G$ will be referred to as the type of $\xi$.

(iii) Similarly, we speak of the sign $\epsilon(T)$ (see 2.3) (resp. $\epsilon(\xi)$ ) of a $\sigma$-stable maximal torus (resp. a semisimple element $\xi \in \mathfrak{G}(\mathbb{R})$ ), the latter being the sign of $T_{\xi}$.

To complete the definitions of terms used in the introduction, we define the index $\epsilon_{0}(G)$ of $G$. For this we choose a Borel subgroup $B_{0}$ containing the fixed maximally split torus $T_{0}$. Note that unless $G$ is quasi-split, there is no Borel subgroup defined 
over $\mathbb{R}$, so $B_{0}$ is not necessarily $\sigma$-stable. Let $v_{0} \in W_{0}$ be the element such that $\sigma\left(B_{0}\right)={ }^{v_{0}} B_{0}$. It is not hard to check that the $\sigma$-conjugacy class of $v_{0}$ does not depend on the choice of $B_{0} \supset T_{0}$. Hence by Lemma 2.2 the following definition is unambiguous.

Definition 2.7. Let $G$ be a reductive group over $\mathbb{R}$. We define the index $\epsilon_{0}(G)$ of $G$ to be the sign $\epsilon\left(v_{0}\right) \in\{-1,1\}$ of the $v_{0} \in W_{0}$ such that $\sigma\left(B_{0}\right)={ }^{v_{0}} B_{0}$ for any maximally split torus $T_{0}$ and any Borel subgroup $B_{0}$ containing $T_{0}$.

\section{Galois cohomology, twisting and $W$-torsors}

In this section we recall some of the terminology and results of [Se, §5], adapted to our situation. We take the group " $G$ " of [Se, loc. cit.] to be $\Gamma=\langle\gamma\rangle$, where $\gamma$ acts as an involutory automorphism on the group $W$, denoted by $w \mapsto \gamma w=\gamma(w)$. Our starting point is therefore a pair $(W, \gamma)$, where $W$ is a finite group and $\Gamma \cong \mathbb{Z} / 2 \mathbb{Z}=\langle\gamma\rangle$ acts on $W$ as an automorphism group. We shall refer to this as a "Galois pair".

3.1. Galois Cohomology. Since $\Gamma=\{1, \gamma\}$, a 1-cocycle $a$ of $\Gamma$ with values in $W$ is determined by its value $a_{\gamma}$ at $\gamma \in \Gamma$. This must satisfy $a_{\gamma^{2}}=a_{\gamma}\left({ }^{\gamma} a_{\gamma}\right)=1$, i.e ${ }^{\gamma} a_{\gamma}=a_{\gamma}^{-1}$, so that $a_{\gamma} \in W_{\gamma}:=\left\{w \in W \mid \gamma(w)=w^{-1}\right\}$. Thus $a$ is determined by $a_{\gamma} \in W_{\gamma}$ and it is easily checked that $w_{1}, w_{2} \in W_{\gamma}$ represent cohomologous cocycles precisely when $w_{2}=v w_{2}^{\gamma} v^{-1}$ for some $v \in W$. We say that $w_{1}, w_{2}$ are $\gamma$-conjugate if they are so related.

The above observation leads to

Lemma 3.1. $\quad$ (i) The set of Galois cocycles $Z^{1}(\Gamma, W)$ of the pair $(W, \gamma)$ is naturally isomorphic to the pointed set $W_{\gamma}$ with the identity of $W$ being the distinguished ('trivial') element.

(ii) The Galois cohomology set $H^{1}(\Gamma, W)$ of the pair $(W, \gamma)$ is naturally isomorphic to the pointed set of $\gamma$-conjugacy classes of $W_{\gamma}$ with the $\gamma$-conjugacy class of the identity being the distinguished ('trivial') element.

Definition 3.2. $\quad$ (i) $A$ (left) $(W, \gamma)$-torsor ( $c f$. [Se, 5.2]), is a finite set $X$ with a regular (left) $W$ action, and a compatible (left) $\Gamma$ action. Thus for $x \in X$ and $w \in W$, we have ${ }^{\gamma}(w \cdot x)=\gamma(w) \cdot \gamma(x)$. When there is no danger of confusion, we shall speak simply of $W$-torsors.

(ii) $A$ pointed (left) $(W, \gamma)$-torsor is a pair $(X, x)$, where $X$ is a (left) $(W, \gamma)$ torsor and $x$ is any point of $X$.

Two $W$-torsors $X, X^{\prime}$ are equivalent if there is a bijection $\phi: X \rightarrow X^{\prime}$ which satisfies the obvious requirements. Similarly, we have an obvious notion of equivalence of pointed $W$-torsors.

If $(X, x)$ is a pointed $W$-torsor, then ${ }^{\gamma} x=v_{(X, x)} \cdot x$ for some (unique) element $v_{(X, x)} \in W$. It is easily verified that $v_{(X, x)} \in W_{\gamma}$, and that the cohomology class of $v_{(X, x)}$ depends only on $X$.

Definition 3.3. For any $W$-torsor $X$ define $[X] \in H^{1}(\Gamma, W)$ by $[X]:=\left(v_{(X, x)}\right)_{\gamma}$, the $\gamma$-conjugacy class of $v_{(X, x)}$, where ${ }^{\gamma} x=v_{(X, x)} \cdot x$ as above. 
Proposition 3.4. $\quad$ (i) ([Se, Proposition 33, p. 47]) The map $X \mapsto[X]$ defined in (3.3) induces a bijection between equivalence classes of $(W, \gamma)$-torsors and the set of cohomology classes $H^{1}(\Gamma, W)$.

(ii) The map $(X, x) \mapsto v_{(X, x)}$ used in (3.3) induces a bijection between equivalence classes of pointed $(W, \gamma)$-torsors and the set of cocycles $Z^{1}(\Gamma, W)$.

Recall that we refer to $(1)_{\gamma} \in H^{1}(\Gamma, W)$ as the trivial cohomology class. The next statement is clear.

Lemma 3.5. The $W$-torsor $X$ corresponds to the trivial cohomology class under the map of Lemma 3.4(i) if and only if $\gamma$ has a fixed point on $X$.

3.2. Galois pairs and group extensions. Let $(W, \gamma)$ be a Galois pair. Consider the semidirect product $\widetilde{W}:=W \rtimes \Gamma$. The element $(1, \gamma) \in \widetilde{W}$ which corresponds to $\gamma \in \Gamma$ will be denoted $\gamma_{W}$, or simply $\gamma$ when there is no risk of confusion. We have an extension of groups

$$
1 \rightarrow W \rightarrow \widetilde{W} \rightarrow \Gamma \rightarrow 1
$$

with a distinguished section that sends $\gamma \in \Gamma$ to $\gamma_{W} \in \widetilde{W}$. Conversely, any extension (3.6) with a section $\psi: \Gamma \rightarrow \widetilde{W}$ defines a Galois pair $(W, \gamma)$, where $\gamma$ acts on $W$ as conjugation by $\psi(\gamma)$.

Let $X$ be a $(W, \gamma)$-torsor. If $x \in X$, then $x \stackrel{\gamma}{\mapsto} \gamma^{\gamma} \stackrel{w}{\mapsto} w \cdot \gamma_{x} \stackrel{\gamma}{\mapsto} \gamma w \cdot x$. Hence among the transformations of $X$ we have the relation $\gamma \circ w \circ \gamma=\gamma w$, from which the next statement is evident.

Lemma 3.7. There is a natural $1-1$ correspondence between (left) $(W, \gamma)$-torsors and (left) $\widetilde{W}$-sets that are regular as $W$-sets.

Corollary 3.8. Let $(W, \gamma)$ and $\left(W^{\prime}, \gamma^{\prime}\right)$ be two Galois pairs. Any isomorphism $\rho: \widetilde{W} \rightarrow \widetilde{W^{\prime}}$ compatible with the projections $\widetilde{W} \rightarrow \Gamma$ and $\widetilde{W}^{\prime} \rightarrow \Gamma$ naturally gives rise to a bijection between the set of $(W, \gamma)$-torsors and the set of $\left(W^{\prime}, \gamma^{\prime}\right)$-torsors.

The corresponding isomorphism

$$
H^{1}(\Gamma, W) \stackrel{\sim}{\rightarrow} H^{1}\left(\Gamma, W^{\prime}\right)
$$

is given by $(v)_{\gamma} \mapsto\left(v_{\rho}^{-1} \rho(v)\right)$, where $v_{\rho} \in W^{\prime}$ is such that $\rho\left(\gamma_{W}\right)=v_{\rho} \gamma_{W^{\prime}}^{\prime}$.

Proof. The first part follows immediately from the preceding lemma; the second part is then easy to verify.

The extension (3.6) comes with the section $\gamma \mapsto \gamma_{W}$. For an arbitrary section $\psi: \Gamma \rightarrow \widetilde{W}$, define $v_{\psi} \in W$ by $\psi(\gamma)=v_{\psi} \gamma_{W}$.

Lemma 3.9. We have $v_{\psi} \in W_{\gamma}$. Further, the map $\psi \mapsto v_{\psi}$ defines a 1-1 correspondence between sections

$$
\psi: \Gamma \rightarrow \widetilde{W}
$$

of the extension (3.6) and cocycles $v_{\psi} \in Z^{1}(\Gamma, W)$.

Proof. Since $\psi(\gamma)$ is an involution, we have $\gamma_{W} v_{\psi} \gamma_{W}=v_{\psi}^{-1}$, proving the first assertion. The bijective nature of this correspondence follows easily. 
Corresponding to the section $\psi: \Gamma \rightarrow \widetilde{W}$, we have the Galois pair $\left(W_{\psi}, \gamma_{\psi}\right)$ in which $\gamma \in \Gamma$ acts on $W$ via the involution $\gamma_{\psi}:=\operatorname{int} v_{\psi} \circ \gamma$, where int $v_{\psi}$ denotes conjugation by $v_{\psi}$.

In general, a twist of the Galois pair $(W, \gamma)$ (cf. [Se]), is a Galois pair of the form $\left(W, \gamma_{v}\right):=(W$, int $v \circ \gamma)$, for some $v \in W_{\gamma}$. Observe that $W$ acts both on the set of Galois pairs $\left(\operatorname{via}(W, \gamma) \stackrel{w}{\longmapsto}\left(W\right.\right.$,int $\left.\left.w^{-1} \circ \gamma \circ \operatorname{int} w\right)\right)$, and on the set of sections $\psi$ of the extension (3.6), by conjugation. In each case the set of equivalence classes may be identified via (3.9) with $H^{1}(\Gamma, W)$. We summarise this in the following extension of (3.9).

Lemma 3.10. Let $(W, \gamma)$ be a Galois pair. The following sets are in canonical bijection:

(i) The twists (resp. equivalence classes of twists) of $(W, \gamma)$.

(ii) The sections (resp. equivalence classes of sections) $\psi$ of the extension (3.6).

(iii) The set $Z^{1}(\Gamma, W)$ (resp. $H^{1}(\Gamma, W)$ ).

Now by Corollary 3.8, the identity map on $\widetilde{W}$ induces an isomorphism

$$
H^{1}(\Gamma, W) \rightarrow H^{1}\left(\Gamma, W_{\psi}\right)
$$

given by $(v)_{\gamma} \mapsto\left(v_{\psi}^{-1} v\right)_{\gamma_{\psi}}$.

The next two lemmas are simple observations, which will be used to relate representations of $\widetilde{W}$ to $W$-torsors.

Lemma 3.12. Let $(X, x)$ be a pointed $(W, \gamma)$-torsor, and let $\widetilde{W}_{x} \leq \widetilde{W}$ be the stabiliser of $x$ in $\widetilde{W}$. Then there is a canonical isomorphism of pointed $(W, \gamma)$-torsors

$$
\left(\widetilde{W} / \widetilde{W}_{x}, \widetilde{W}_{x}\right) \stackrel{\sim}{\rightarrow}(X, x) .
$$

In particular $\gamma_{W} \widetilde{W}_{x}=v_{(X, x)} \widetilde{W}_{x}$.

To identify the stabiliser groups, we have

Lemma 3.13. Let $(X, x)$ be a pointed $(W, \gamma)$-torsor with corresponding cocycle $v_{(X, x)} \in Z^{1}(\Gamma, W)$ (Def. (3.2)). Then $\widetilde{W}_{x}=\psi(\Gamma)$, where $\psi: \Gamma \rightarrow \widetilde{W}$ is the section corresponding to $v_{(X, x)}^{-1}$ (Lemma (3.9)).

We apply this to give a convenient way of describing the cohomology of a torsor (considered as a topological space endowed with the discrete topology). Here we take coefficients in an arbitrary field $\mathbb{F}$ of characteristic zero.

Corollary 3.14. Let $(X, x)$ be a pointed $W$-torsor. Let $\widetilde{W}_{x} \subset \widetilde{W}$ be the stabiliser of $x$. Then the $\widetilde{W}$-representation

$$
H^{0}(X, \mathbb{F})=\bigoplus_{y \in X} \mathbb{F}
$$

is isomorphic to the induced representation $\operatorname{Ind} \frac{\widetilde{W}}{W_{x}} \mathbb{F}$.

We shall use the following explicit calculation in $\S 5$ in the proof of Lemma 5.2. 
Lemma 3.15. Let $(X, x)$ be a pointed $(W, \gamma)$-torsor. Let $\alpha: \widetilde{W} \rightarrow \mathbb{F}^{*}$ be a 1dimensional representation of $\widetilde{W}$ in which the element $\gamma_{W} \in \widetilde{W}$ acts trivially. Then the $\Gamma$-module $\operatorname{Hom}_{W}\left(\alpha, H^{0}(X, \mathbb{F})\right)$ has dimension 1 , and $\gamma$ acts as $\alpha\left(v_{(X, x)}\right)$, where $v_{(X, x)}$ is the cocycle corresponding to $(X, x)$ considered as an element of $W_{\gamma} \subset W$.

Proof. (Compare the proof of [Le2, Lemma 7.12].) Observe that since $H^{0}(X, \mathbb{F})$ is just the regular $W$-module, $\operatorname{Hom}_{W}\left(\alpha, H^{0}(X, \mathbb{F})\right)$ is one-dimensional, with basis element $\sum_{v \in W} \alpha(v)^{-1} v \widetilde{W}_{x}$, where the basis elements of $H^{0}(X, \mathbb{F})$ are identified (3.14) with cosets of $\widetilde{W}_{x}$. By the last sentence in Lemma 3.12, $\gamma_{W} \sum_{v \in W} \alpha(v)^{-1} v \widetilde{W}_{x}=\sum_{v \in W} \alpha(v)^{-1} \gamma(v) \gamma_{W} \widetilde{W}_{x}=\alpha\left(v_{(X, x)}\right) \sum_{v \in W} \alpha(v)^{-1} \gamma(v) \widetilde{W}_{x}$, which equals $\alpha\left(v_{(X, x)}\right) \sum_{v \in W} \alpha(v)^{-1} v \widetilde{W}_{x}$ by the hypothesis on $\alpha$.

3.3. Unramified coverings and the absolute Weyl group. Let $p: \mathcal{P} \rightarrow \mathcal{T}$ be an arbitrary unramified Galois covering of nonsingular complex algebraic varieties defined over $\mathbb{R}$ (i.e., $\mathcal{P}, \mathcal{T}$ and $p$ are all defined over $\mathbb{R}$ ). Let $W$ be the Galois group of covering transformations of the covering $p: \mathcal{P} \rightarrow \mathcal{T}$. Then $\Gamma:=\operatorname{Gal}(\mathbb{C} / \mathbb{R})=\langle\sigma\rangle$ acts via antiholomorphic involutions $\sigma_{\mathcal{P}}$ and $\sigma_{\mathcal{T}}$ respectively on $\mathcal{P}$ and $\mathcal{T}$, compatibly (i.e. commuting) with the projection $p$. Hence the involution $\sigma_{\mathcal{P}}$ normalises the group $W$ of covering transformations, and the semidirect product $\widetilde{W}=\left\langle W, \sigma_{\mathcal{P}}\right\rangle$ acts on $\mathcal{P}$ as a group of transformations respecting the projection $\mathcal{P} \rightarrow \mathcal{T} / \Gamma$.

Remark 3.16. Readers familiar with orbifold theory will recognise $\widetilde{W}$ as the Galois group of the covering $\mathcal{P} \rightarrow \mathcal{T} / \Gamma$ of the orbifold $\mathcal{T} / \Gamma$; readers familiar with Galois theory of schemes will recognize this as the Galois group of the covering $\mathcal{P} \times \operatorname{Spec} \mathbb{R}$ Spec $\mathbb{C} \rightarrow \mathcal{T}$ of the $\mathbb{R}$-scheme $\mathcal{T}$.

The covering $p: \mathcal{P} \rightarrow \mathcal{T}$ defines an exact sequence

$$
1 \rightarrow W \rightarrow \widetilde{W} \rightarrow \Gamma \rightarrow 1
$$

with a section $\Gamma \rightarrow \widetilde{W}$ given by $\sigma \mapsto \sigma_{\mathcal{P}}$, and an action of $\Gamma$ on $W$ given by $\sigma \cdot w=$ $\sigma_{\mathcal{P}} \circ w \circ \sigma_{\mathcal{P}}$. This data defines a Galois pair $(W, \gamma)$ as in $\S 3$.

Definition 3.18. $A$ (Galois) twist of the covering $p: \mathcal{P} \rightarrow \mathcal{T}$ is a covering $p^{\prime}: \mathcal{P}^{\prime} \rightarrow$ $\mathcal{T}$, where $\mathcal{P}^{\prime}$ is a complex variety with real structure corresponding to an involution $\sigma_{\mathcal{P}^{\prime}}$ and $p^{\prime}$ commutes with the real structures $\sigma_{\mathcal{P}^{\prime}}$ and $\sigma_{\mathcal{T}}$ on $\mathcal{P}^{\prime}$ and $\mathcal{T}$ respectively, such that there is an isomorphism of complex varieties $\phi: \mathcal{P} \rightarrow \mathcal{P}^{\prime}$ with $p=p^{\prime} \circ \phi$. The isomorphism $\phi$ canonically identifies the groups $W$ and $\widetilde{W}$ with $W^{\prime}$ and $\widetilde{W}^{\prime}$ respectively, since $W=\phi^{-1} W^{\prime} \phi$, etc. We say that $p^{\prime}: \mathcal{P}^{\prime} \rightarrow \mathcal{T}$ is equivalent to $p: \mathcal{P} \rightarrow \mathcal{T}$ if $\phi$ can be chosen so that $\phi^{-1} \sigma_{\mathcal{P}^{\prime}} \phi=\sigma_{\mathcal{P}}$. This clearly defines an equivalence relation on the set of Galois twists.

Proposition 3.19. (cf. (3.10)) There is a bijection between the set of equivalence classes of twists of the covering $\mathcal{P} \rightarrow \mathcal{T}$ and the Galois cohomology set $H^{1}(\Gamma, W)$.

Proof. Any section $\psi: \Gamma \rightarrow \widetilde{W}$ of the extension (3.17) gives rise to a an antihomolomorphic involution $\sigma_{\psi}=\psi(\sigma)$ on $\mathcal{P}$, hence to a different real structure on $\mathcal{P}$ which still commutes with the projection $p$ and the real structure of $\mathcal{T}$. We therefore obtain 
a covering $p^{\prime}: \mathcal{P}^{\prime} \rightarrow \mathcal{T}$, where $p^{\prime}=p$ and $\mathcal{P}^{\prime}=\mathcal{P}$, but with the new real structure $\sigma_{\psi}$. This is a twist (see (3.18)) of the original covering, and since $W$ is the group of all automorphisms of the covering $p, \psi$ and $\psi^{\prime}$ give equivalent twists if and only if they are conjugate in $W$.

By Lemma 3.10, it remains only to show that every twist is equivalent to one arising from a section as above. If $\mathcal{P}^{\prime} \rightarrow \mathcal{T}$ is any Galois twist of the covering $p$, then there is an isomorphism $\phi: \mathcal{P} \stackrel{\sim}{\rightarrow} \mathcal{P}^{\prime}$, which defines a section $\Gamma \rightarrow \widetilde{W}$ given by $\psi: \sigma \mapsto \sigma^{\prime}=\phi^{-1} \circ \sigma_{\mathcal{P}^{\prime}} \circ \phi \in \widetilde{W}$. It is easily checked that $\mathcal{P}^{\prime} \rightarrow \mathcal{T}$ is equivalent to $p$, with $\mathcal{P}$ having real structure given by $\sigma^{\prime}=\psi(\sigma)$.

Now take $\mathcal{P}$ and $\mathcal{T}$ as in $\S 1$. Then, as the notation suggests, the Galois group $W$ can be considered as the 'absolute' Weyl group of $G$. Indeed, any pair $\left(T, B_{T}\right)$ consisting of a maximal torus $T \subset G$ defined over $\mathbb{R}$ and a Borel group $B_{T} \subset G$ containing $T$ determines isomorphisms $G / N_{G}(T) \stackrel{\sim}{\rightarrow} \mathcal{T}$ and $G / T \stackrel{\sim}{\rightarrow} \mathcal{P}$ of complex algebraic varieties defined by sending the coset $g N_{G}(T) \in G / N_{G}(T)$ (resp. $g T \in G / T$ ) to the torus ${ }^{g} T$ (resp. the pair $\left.\left({ }^{g} T,{ }^{g} B_{T}\right) \in \mathcal{P}\right)$. These isomorphisms are clearly compatible with the covering projections $G / T \rightarrow G / N_{G}(T)$ and $\mathcal{P} \rightarrow \mathcal{T}$. The isomorphism $G / N_{G}(T) \stackrel{\sim}{\rightarrow} \mathcal{T}$ is clearly defined over $\mathbb{R}$. However in general there may be no $B_{T}$ which is defined over $\mathbb{R}$. Thus the natural covering $G / T \rightarrow G / N_{G}(T)$ of complex algebraic varieties defined over $\mathbb{R}$ is a twist (see (3.18)) of the covering $\mathcal{P} \rightarrow \mathcal{T}$. Now the Galois group of the covering $G / T \rightarrow G / N_{G}(T)$ is canonically isomorphic to $W_{T}=N_{G}(T) / T$, with the $\Gamma$-action on $N_{G}(T) / T$ corresponding to conjugation by the antiholomorphic involution $\sigma_{G / T}$ of $G / T$. Hence our choice of $B_{T}$ determines isomorphisms $W \simeq W_{T}$ and $\widetilde{W} \simeq \widetilde{W_{T}}$. If $B_{T}$ is replaced by $B_{T}^{\prime}$, then $B_{T}^{\prime}=\operatorname{int} w B_{T}$ for some element $w \in W_{T}$. Hence the isomorphism $G / T \rightarrow \mathcal{P}$ is composed with an element of the Galois group of the covering $\mathcal{P} \rightarrow \mathcal{T}$, and the isomorphism $W \simeq W_{T}$ is composed with conjugation by $w \in W_{T}$.

This discussion is summarised in the next result.

Lemma 3.20. Let $T$ be a $\sigma$-stable maximal torus of $G$, and write $W_{T}$ for the Weyl group of $G$ with respect to $T$. Then

(i) There is a canonical isomorphism, uniquely determined by $T$, of $\Gamma$-varieties $G / N_{G}(T) \simeq \mathcal{T}$.

(ii) Any Borel subgroup $B_{T} \supseteq T$ determines an isomorphism of complex varieties $G / T \stackrel{\phi_{T}}{\longrightarrow} \mathcal{P}$. This isomorphism is uniquely determined by $T$ up to composition with an element of the Galois group $W$ of $\mathcal{P} \rightarrow \mathcal{T}$.

(iii) If $p_{T}: G / T \rightarrow \mathcal{T} \simeq G / N_{G}(T)$ is the covering arising from the identification (i), then $p_{T}=p \circ \phi_{T}$ for any choice of $\phi_{T}$. Thus $p_{T}$ is a well-defined Galois twist of $p$.

(iv) (cf. (3.18)) The isomorphism $\phi_{T}$ defines isomorphisms $W_{T} \stackrel{\sim}{\rightarrow} W$ and $\widetilde{W_{T}} \rightarrow$ $\widetilde{W}$. These isomorphisms are uniquely defined by $T$, up to (composition with) conjugation by an element of $W_{T}$. 
We are particularly interested in the case where $T=T_{0}$ as in $\S 2$. We then write $B_{0}:=B_{T_{0}}, W_{0}:=W_{T}$, etc. We will identify $G / N_{G}\left(T_{0}\right)$ and $\mathcal{T}$.

Remark 3.21. We have adopted the convention that Galois groups of Galois coverings act on the left. This means that the Galois group $W_{T}=N_{G}(T) / T$ acts on $G / T$ by $w \cdot g T=g w^{-1} T$.

Lemma 3.22. With notation as above, we have

(i) The coverings $p: \mathcal{P} \rightarrow \mathcal{T}$ and $p_{0}: G / T_{0} \rightarrow \mathcal{T}$ are twists of each other (3.18).

(ii) The two coverings are isomorphic over $\mathbb{R}$ if and only if $G$ is quasi-split.

(iii) Under the isomorphism $\widetilde{W} \simeq \widetilde{W}_{0}$ determined by $B_{0}, \sigma_{\mathcal{P}}=v_{0}^{-1} \sigma_{G / T_{0}}$, where $v_{0} \in W_{0}$ is such that $\sigma\left(B_{0}\right)={ }^{v_{0}} B_{0}$.

(iv) The class in $H^{1}\left(\Gamma, W_{0}\right)$ corresponding to the twist $\mathcal{P} \rightarrow \mathcal{T}$ of the covering $G / T_{0} \rightarrow \mathcal{T}$ is represented by the cocycle $v_{0}^{-1}$ defined above.

Proof. This is all clear from the above discussion, provided we know that if $G$ is quasisplit then $G$ has a $\sigma$-stable Borel subgroup containing $T_{0}$. This last assertion may be justified as follows. Let $B$ be a $\sigma$-stable Borel subgroup of $G$. Then $B$ contains a maximal torus $T$ which is $\sigma$-stable (see [BT, Théorème 2.14(i)]), and clearly the unipotent radical $U$ of $B$ is $\sigma$-stable. It follows from [BT, Corollaire 3.18, p.82] that $U$ is split over $\mathbb{R}$, from which it follows that $T=S_{0} Z$, where $S_{0}$ is a split torus of rank equal to the semisimple rank of $G$, and $Z=Z(G)$, which is also $\sigma$-stable. It follows that if $Z_{s}$ is the split part of $Z$, then $S=S_{0} Z_{s}$ is a maximal split torus in $G$. Thus $T$ is $G(\mathbb{R})$-conjugate to $T_{0}$, and the assertion follows.

Corollary 3.23. Let $G$ be a connected reductive group defined over $\mathbb{R}$. The index $\epsilon_{0}(G)$ of $G$ with respect to the maximally split maximal torus $T_{0}$ (as defined in (2.7)) equals the sign of the class in $H^{1}\left(\Gamma, W_{0}\right)$ of the twist $\mathcal{P} \rightarrow \mathcal{T}$ of the covering $G / T_{0} \rightarrow \mathcal{T}$.

Remark 3.24. Similarly, for any $T \in \mathcal{T}(\mathbb{R})$, the sign $\epsilon(T)$ coincides with the sign of the class in $H^{1}\left(\Gamma, W_{0}\right)$ of the fibre of the covering $G / T_{0} \rightarrow \mathcal{T}$ at $T \in \mathcal{T}(\mathbb{R})$, which in turn coincides with the sign of the cohomology class in $H^{1}\left(\Gamma, W_{0}\right)$ associated to the twist $G / T \rightarrow \mathcal{T}$ of the covering $G / T_{0} \rightarrow \mathcal{T}$.

\section{LEFSCHETZ NUMBERS AND WEIGHTED EULER CHARACTERISTICS}

In this section we prove the Lefschetz trace formula for local systems on quasiprojective varieties over $\mathbb{R}$. In particular, we make a connection between Lefschetz numbers for local systems and "weighted Euler characteristics".

Let us fix a field $\mathbb{F}$ of characteristic zero. The Lefschetz trace formula for endomorphisms of compact topological manifolds yields the following formula for a nonsingular projective variety $X$ over $\mathbb{R}$ :

$$
\sum_{i}(-1)^{i} \operatorname{Trace}\left(\sigma, H^{i}(X(\mathbb{C}), \mathbb{F})\right)=\chi(X(\mathbb{R})),
$$

where $\chi$ denotes the ordinary Euler characteristic. Since the spaces $\mathcal{T}_{\xi}(\mathbb{C})$ and $\mathcal{T}^{\xi}(\mathbb{C})$ are not compact (and possibly singular), the more general formula that we need, no longer follows from a statement about arbitrary endomorphisms. Instead, we will use 
the fact that we actually have an involution and an equivariant simplicial decomposition.

Definition 4.1. Let $\mathbb{F}$ be a field of characteristic zero. Let $X$ be a finite dimensional locally compact topological space with an action of $\Gamma=\mathbb{Z} / 2=\{1, \sigma\}$. A $\Gamma$-equivariant sheaf of $\mathbb{F}$-vector spaces on $X$ is a sheaf $\mathcal{S}$ of $\mathbb{F}$-vector spaces together with a collection of isomorphisms

$$
\left\{\alpha_{g}: \mathcal{S} \stackrel{\sim}{\rightarrow} g^{*} \mathcal{S}\right\}_{g \in \Gamma}
$$

such that $\alpha_{1}$ is the identity map and $\alpha_{g h}=h^{*}\left(\alpha_{g}\right) \circ \alpha_{h}$ for all $g, h \in \Gamma$.

Since $\Gamma$ is generated by $\sigma$, we shall use the terminology ' $\sigma$-equivariant' interchangeably with ' $\Gamma$-equivariant'.

Definitions 4.2. Let $\mathcal{V}$ be a locally constant $\Gamma$-equivariant sheaf of $\mathbb{F}$-vector spaces on $X(\mathbb{C})$ (i.e., a local system).

(i) The Lefschetz number of $\sigma$ acting on $(X, \mathcal{V})$ is the alternating sum of the traces:

$$
\Lambda_{\mathrm{c}}(\sigma, X, \mathcal{V}):=\sum_{i}(-1)^{i} \operatorname{Trace}\left(\sigma, H_{c}^{i}(X, \mathcal{V})\right) .
$$

Here we assume that all cohomology groups occurring in the sum are finitedimensional.

(ii) For any two points $x, y$ in the same path connected component of the fixed point space $X^{\sigma}:=\{x \in X \mid \sigma(x)=x\}$, the stalks $\mathcal{V}_{x}$ and $\mathcal{V}_{y}$ are isomorphic as $\Gamma$-representations and we write $\mathcal{V}_{C}$ for the equivalence class of the representations $\mathcal{V}_{x}$.

(iii) The weighted Euler characteristic of $\left(X^{\sigma}, \mathcal{V}\right)$ will be the sum

$$
\sum_{\substack{C \subset X^{\sigma} \\ \text { connected } \\ \text { component }}} \chi_{c}(C) \cdot \operatorname{Trace}\left(\sigma, \mathcal{V}_{C}\right) .
$$

Here $\chi_{c}(C)$ is the compact support Euler characteristic $\sum_{i}(-1)^{i} \operatorname{dim} H_{c}^{i}(C, \mathbb{F})$, always assuming this sum is finite.

We shall show that the weighted Euler characteristic is equal to the Lefschetz number $\Lambda_{\mathrm{c}}(\sigma, X, \mathcal{V})$.

4.1. The trace formula for involutions of simplicial complexes. Suppose we have a finite simplicial complex $\mathfrak{K}$ and a simplicial subcomplex $\mathfrak{L} \subset \mathfrak{K}$, such that our topological space $X$ has the form

$$
X=|\mathfrak{K}|-|\mathfrak{L}|
$$

and such that the involution $\sigma: X \rightarrow X$ comes from a simplicial automorphism of $\mathfrak{K}$. We will denote this simplicial endomorphism by $\sigma$ as well. Possibly after replacing $\mathfrak{K}$ by its barycentric subdivision, we may assume that any simplex stable under $\sigma$ is actually pointwise fixed by $\sigma$ (see [Br, §III.1]). This implies in particular that the fixed point set of $\sigma$ is a simplicial subcomplex of $\mathfrak{K}$ : $\left|\mathfrak{K}^{\sigma}\right|=|\mathfrak{K}|^{\sigma}$. 
Proposition 4.3. Let $X$ and $\sigma$ be as above. Let $\mathcal{V}$ be a $\sigma$-equivariant locally constant sheaf of $\mathbb{F}$-vector spaces on $X$. We have

$$
\Lambda_{\mathrm{c}}(\sigma, X, \mathcal{V})=\Lambda_{\mathrm{c}}\left(\left.\sigma\right|_{X^{\sigma}}, X^{\sigma},\left.\mathcal{V}\right|_{X^{\sigma}}\right)
$$

$$
\Lambda_{\mathrm{c}}\left(\left.\sigma\right|_{X^{\sigma}}, X^{\sigma},\left.\mathcal{V}\right|_{X^{\sigma}}\right)=\sum_{\begin{array}{c}
C \subset X^{\sigma} \\
\text { connected } \\
\text { component }
\end{array}} \chi_{c}(C) \cdot \operatorname{Trace}\left(\sigma, \mathcal{V}_{C}\right)
$$

Proof. (i) Putting $U=X-X^{\sigma}$, it follows from the excision long exact sequence

$$
\cdots \rightarrow H_{c}^{i}\left(U,\left.\mathcal{V}\right|_{U}\right) \rightarrow H_{c}^{i}(X, \mathcal{V}) \rightarrow H_{c}^{i}\left(X^{\sigma},\left.\mathcal{V}\right|_{X^{\sigma}}\right) \rightarrow \cdots
$$

that the Lefschetz trace can be written as a sum

$$
\Lambda_{\mathrm{c}}(\sigma, X, \mathcal{V})=\Lambda_{\mathrm{c}}\left(\left.\sigma\right|_{U}, U,\left.\mathcal{V}\right|_{U}\right)+\Lambda_{\mathrm{c}}\left(\left.\sigma\right|_{X^{\sigma}}, X^{\sigma},\left.\mathcal{V}\right|_{X^{\sigma}}\right)
$$

Hence it is sufficient to prove that $\Lambda_{\mathrm{c}}\left(\left.\sigma\right|_{U}, U,\left.\mathcal{V}\right|_{U}\right)=0$.

Let $X=|\mathfrak{K}|-|\mathfrak{L}|$ be as above. For any $i$, let $\mathfrak{K}_{i}$ be the union of simplices of $\mathfrak{K}$ of dimension $\leq i$, and let

$$
\left|\mathfrak{K}_{i}\right|^{\circ}:=\left|\mathfrak{K}_{i}\right|-\left|\mathfrak{K}_{i-1}\right|
$$

be the 'interior' of $\left|\mathfrak{K}_{i}\right|$. We write

$$
\begin{aligned}
U_{i} & :=U \cap\left|\mathfrak{K}_{i}\right|, \\
U_{i}^{\circ} & :=U \cap\left|\mathfrak{K}_{i}\right|^{\circ} .
\end{aligned}
$$

Using the excision long exact sequence as above, we see by induction that we have

$$
\Lambda_{\mathrm{c}}\left(\left.\sigma\right|_{U}, U,\left.\mathcal{V}\right|_{U}\right)=\sum_{i=0}^{n} \Lambda_{\mathrm{c}}\left(\left.\sigma\right|_{U_{i}^{\circ}}, U_{i}^{\circ},\left.\mathcal{V}\right|_{U_{i}^{\circ}}\right) .
$$

Each $U_{i}^{\circ}$ is a finite disjoint union of open $i$-simplices, and $\sigma$ permutes them without fixing any connected component. It follows that $\Lambda_{\mathrm{c}}\left(\left.\sigma\right|_{U_{i}^{\circ}}, U_{i}^{\circ},\left.\mathcal{V}\right|_{U_{i}^{\circ}}\right)=0$. Taking the sum over all $i$ we obtain $\Lambda_{\mathrm{c}}\left(\left.\sigma\right|_{U}, U,\left.\mathcal{V}\right|_{U}\right)=0$, hence $\Lambda_{\mathrm{c}}(\sigma, X, \mathcal{V})=\Lambda_{\mathrm{c}}\left(\left.\sigma\right|_{X^{\sigma}}, X^{\sigma},\left.\mathcal{V}\right|_{X^{\sigma}}\right)$.

(ii) It is sufficient to prove that for any connected component $C \subset X^{\sigma}$ we have

$$
\Lambda_{\mathrm{c}}\left(\left.\sigma\right|_{C}, C,\left.\mathcal{V}\right|_{C}\right)=\chi(C) \cdot \operatorname{Trace}\left(\sigma, \mathcal{V}_{C}\right)
$$

With notation as above, we have by excision that

$$
\Lambda_{\mathrm{c}}\left(\left.\sigma\right|_{C}, C,\left.\mathcal{V}\right|_{C}\right)=\sum_{i \geq 0} \Lambda_{\mathrm{c}}\left(\left.\sigma\right|_{C_{i}^{\circ}}, C_{i}^{\circ},\left.\mathcal{V}\right|_{C_{i}^{\circ}}\right)
$$

For any $i \geq 0$ the space $C_{i}^{\circ}$ is a disjoint union of of copies of the open simplices $\Delta_{i}^{\circ}$. Since $\Delta_{i}^{\circ}$ is contractible, the restriction of $\mathcal{V}$ to any connected component of $C_{i}^{\circ}$ is $\Gamma$-equivariantly isomorphic to the $\Gamma$-equivariant constant sheaf associated to the representation $\mathcal{V}_{C}$. By definition $\operatorname{Trace}\left(\sigma, \mathcal{V}_{x}\right)=\operatorname{Trace}\left(\sigma, \mathcal{V}_{C}\right)$ for any $x \in C$, this implies that for any $q, i \geq 0$ we have

$$
\operatorname{Trace}\left(\sigma, H_{c}^{q}\left(C_{i}^{\circ},\left.\mathcal{V}\right|_{C_{i}^{\circ}}\right)\right)=\operatorname{dim} H_{c}^{q}\left(C_{i}^{\circ}, \mathbb{F}\right) \cdot \operatorname{Trace}\left(\sigma, \mathcal{V}_{C}\right) \text {. }
$$

Hence

$$
\Lambda_{\mathrm{c}}\left(\left.\sigma\right|_{C}, C,\left.\mathcal{V}\right|_{C}\right)=\chi_{c}(C) \cdot \operatorname{Trace}\left(\sigma, \mathcal{V}_{C}\right)
$$


by excision.

Remark 4.4. The above approach is straightforward and has been used before (see for example [Kaw91, Th. 5.59] for the case where $X$ is a compact simplicial complex and the local system $\mathcal{V}$ is trivial), but in this generality we have not been able to find the result in the literature.

Theorem 4.5. Let $X$ be a quasi-projective algebraic variety over $\mathbb{R}$, and let $\mathcal{V}$ be a $\sigma$-equivariant locally constant sheaf of $\mathbb{F}$-vector spaces on $X(\mathbb{C})$. Then

$$
\Lambda_{\mathrm{c}}(\sigma, X(\mathbb{C}), \mathcal{V})=\sum_{\substack{C \subset X(\mathbb{R}) \\ \text { connected } \\ \text { component }}} \chi_{c}(C) \cdot \operatorname{Trace}\left(\sigma, \mathcal{V}_{C}\right),
$$

Proof. This is a special case of Proposition 4.3. In order to show that $X(\mathbb{C})$ is the difference between two simplicial complexes with a $\sigma$-action, we observe that $X(\mathbb{C})$ has a natural semi-algebraic structure and that the antiholomorphic involution $\sigma$ is semialgebraic itself. It is then a fairly straightforward consequence of the triangulisability of semi-algebraic sets, originally due to Eojasiewicz ([E], see also $[\mathrm{H}]$ ) that we have a finite simplicial complex $\mathfrak{K}$ with a $\sigma$-action and a $\sigma$-equivariant subcomplex $\mathfrak{L} \subset \mathfrak{K}$ such that $X(\mathbb{C})$ is equivariantly homeomorphic to $|\mathfrak{K}|-|\mathfrak{L}|$; see for example [PS, Th. 4.5, Th. 4.6]. Hence Proposition 4.3 applies.

\section{The VARIETIES $\mathcal{T}^{\xi}$}

We shall deal in this section with the varieties $\mathcal{T}^{\xi}$, defined in 1.5. Our basic strategy is to effectively reduce their study to the case $\xi=0$.

Lemma 5.1. Let $G, \mathfrak{G}$ etc. be as in $\S 1$, and let $\xi$ be a semisimple element in $\mathfrak{G}(\mathbb{R})$. Let $Z_{G}(\xi)^{0}$ be the connected component of the identity in the centraliser $Z_{G}(\xi)$ of $\xi$ in the adjoint representation. Then in the obvious notation, we have a canonical isomorphism of $\Gamma$-varieties, $\mathcal{T}^{\xi}(G) \stackrel{\sim}{\longrightarrow} \mathcal{T}\left(Z_{G}(\xi)^{0}\right)$, where the Galois action on the reductive $\mathbb{R}$-group $Z_{G}(\xi)^{0}$ is the restriction of the Galois action on $G$.

Proof. Observe first that by [Spr, 11.2.8], $Z_{G}(\xi)^{0}$ is a reductive group which is defined over $\mathbb{R}$. Next, a maximal torus $T \in \mathcal{T}$ satisfies $\operatorname{Lie} T \ni \xi$ (i.e. is in $\mathcal{T}^{\xi}$ ) if and only if Lie $T$ is contained in the centraliser $Z_{\mathfrak{G}}(\xi)$. But by [Bo, pp. 225 and 321], $Z_{\mathfrak{G}}(\xi)=\operatorname{Lie} Z_{G}(\xi)^{0}$ and hence $T \in \mathcal{T}^{\xi}$ if and only if $T$ is contained in $Z_{G}(\xi)^{0}$. Thus the varieties $\mathcal{T}^{\xi}(G)$ and $\mathcal{T}\left(Z_{G}(\xi)^{0}\right)$ are equal, with the $\Gamma$-action on the latter being the restriction of the action on the former.

We next wish to relate the Lefschetz numbers of $\sigma$ acting on the cohomology of the two local systems on $\mathcal{T}^{\xi}$ which arise from the identification (5.1), viz. $\mathcal{S}_{\epsilon}=\mathcal{S}_{\epsilon}(G)$ above, and the local system $\mathcal{S}_{\epsilon}\left(Z_{G}(\xi)^{0}\right)$ on $\mathcal{T}\left(Z_{G}(\xi)^{0}\right)$ obtained by considering the case " $\xi=0$ " for the group $Z_{G}(\xi)^{0}$.

Lemma 5.2. The trace Trace $\left(\sigma, \mathcal{S}_{\epsilon, T}\right)$ of $\sigma$ on the stalk $\mathcal{S}_{\epsilon, T}$ at $T \in \mathcal{T}(\mathbb{R})$ of the local system $\mathcal{S}_{\epsilon}$ is $\epsilon_{0}(G) \epsilon(T)$, where $\epsilon_{0}(G)$ is the sign defined in $(2.7)$ and $\epsilon(T)$ is the sign of $T$ as defined in (2.6). 
Proof. On the one hand, it follows from the definitions that $\epsilon(T)$ is the sign of the Galois cohomology class in $H^{1}\left(\Gamma, W_{0}\right)$ of the fibre at $T$ of the covering $G / T_{0} \rightarrow \mathcal{T}$. On the other hand, we have by Lemma 3.15, that $\operatorname{Trace}\left(\sigma, \mathcal{S}_{\epsilon, T}\right)$ is the sign of the Galois cohomology class in $H^{1}(\Gamma, W)$ of the fibre at $T$ of the covering $\mathcal{P} \rightarrow \mathcal{T}$. Hence the statement follows from Corollary 3.8 and Lemma 3.22.

Proposition 5.3. Maintain notation as in (5.1), and let $\xi$ be a semisimple element in $\mathfrak{G}(\mathbb{R})$. Then

$$
\Lambda_{\mathrm{c}}\left(\sigma, \mathcal{T}^{\xi}(G), \mathcal{S}_{\epsilon}(G)\right)=\epsilon_{0}(G) \epsilon_{0}\left(Z_{G}(\xi)^{0}\right) \epsilon\left(v_{\xi}\right) \Lambda_{\mathrm{c}}\left(\sigma, \mathcal{T}\left(Z_{G}(\xi)^{0}\right), \mathcal{S}_{\epsilon}\left(Z_{G}(\xi)^{0}\right)\right),
$$

where $v_{\xi} \in W_{0}$ is the type (see (2.6) of a maximally split torus in $Z_{G}(\xi)^{0}$.

Proof. Let $L=Z_{G}(\xi)^{0}$. By (4.5) and (5.1), we need to compare, for any torus $T \in$ $\mathcal{T}(L)^{\sigma}$, the values of $\operatorname{Trace}\left(\sigma, \mathcal{S}_{\epsilon}(G)_{T}\right)$ and $\operatorname{Trace}\left(\sigma, \mathcal{S}_{\epsilon}(L)_{T}\right)$.

For any element $w \in W_{0}$, we use the notation $\dot{w}$ to indicate an element in $N_{0} \subset G$ which represents the coset $w$ of $T_{0}$. Let $T_{\xi}$ be a maximally split torus of $L$ and suppose that $g \in G$ and $v=v_{\xi} \in W_{0}$ are such that $T_{\xi}={ }^{g} T_{0}$ and $g^{-1} \sigma(g)=\dot{v}$. Note that the Weyl group $W\left(G, T_{\xi}\right)$ may be identified with ${ }^{g} W_{0}$, and $W\left(L, T_{\xi}\right)$ with a parabolic subgroup of the former.

Now $T={ }^{l} T_{\xi}$, for some element $l \in L$, and $l^{-1} \sigma(l)=g \dot{w}_{L} g^{-1} \in W\left(L, T_{\xi}\right)$, for some element $w_{L} \in W_{0}$. By (5.2) applied to $(L, \sigma)$, it follows that

$$
\operatorname{Trace}\left(\sigma, \mathcal{S}_{\epsilon}(L)_{T}\right)=\epsilon_{0}(L) \epsilon\left(w_{L}\right) .
$$

Further, $T={ }^{l} T_{\xi}={ }^{l g} T_{0}$, and $(l g)^{-1} \sigma(l g)=g^{-1} g \dot{w}_{L} g^{-1} \sigma(g)=\dot{w}_{L} \dot{v}_{\xi}$. Hence again by $(5.2)$,

$$
\operatorname{Trace}\left(\sigma, \mathcal{S}_{\epsilon}(G)_{T}\right)=\epsilon_{0}(G) \epsilon\left(w_{L}\right) \epsilon\left(v_{\xi}\right) .
$$

Combining (5.5) with (5.6), we therefore obtain, for any maximal torus $T \in \mathcal{T}^{\xi}(\mathbb{R})$,

$$
\operatorname{Trace}\left(\sigma, \mathcal{S}_{\epsilon}(G)_{T}\right)=\epsilon_{0}(G) \epsilon_{0}(L) \epsilon\left(v_{\xi}\right) \operatorname{Trace}\left(\sigma, \mathcal{S}_{\epsilon}(L)_{T}\right) .
$$

Now (5.4) follows immediately from (4.5) and (5.1), applied respectively to $G$ and to $L$.

Proposition 5.8. The value of the Lefschetz number of $\mathcal{S}_{\epsilon}$ is given by

$$
\Lambda_{\mathrm{c}}\left(\sigma, \mathcal{T}, \mathcal{S}_{\epsilon}\right)=\operatorname{Trace}\left(\sigma, H_{c}^{2 N}(G / T, \mathbb{F})\right)=(-1)^{N}
$$

where $N$ is the number of positive roots of $G$.

Proof. By definition, $\Lambda_{\mathrm{c}}\left(\sigma, \mathcal{T}, \mathcal{S}_{\epsilon}\right)$ is equal to $\sum_{j}(-1)^{j} \operatorname{Trace}\left(\sigma, H_{c}^{j}(\mathcal{P}, \mathbb{F})^{\epsilon}\right)$, where the superscript denotes the $\epsilon$-isotypic component. Using (3.22)(i), it follows from the corresponding statement for $G / T_{0}$ ([Le1, Cor. 1.9]), which uses the minimally pure nature of $G / T_{0}$, that

$$
H_{c}^{i}(\mathcal{P}, \mathbb{F})^{\epsilon}=\left\{\begin{array}{l}
H_{c}^{i}(\mathcal{P}, \mathbb{F}) \cong \mathbb{F} \text { if } i=2 N \\
0 \text { otherwise },
\end{array}\right.
$$

Since $\sigma$ is an involution, it acts as \pm 1 on $H_{c}^{2 N}(\mathcal{P}, \mathbb{F})$. 
To determine the sign, observe that there is a $\sigma$-equivariant fibration $\mathcal{P} \longrightarrow \mathcal{B}$ given by $(T \subseteq B) \mapsto B$, which has fibre $U \cong \mathbb{A}^{N}$, where $N$ is the number of positive roots of $G$. Hence $H_{c}^{2 N}(\mathcal{P}, \mathbb{F}) \cong H_{c}^{0}(\mathcal{B}, \mathbb{F}) \otimes H_{c}^{2 N}\left(\mathbb{A}^{N}, \mathbb{F}\right)$. Now $\sigma$ clearly acts trivially on the first factor $H_{c}^{0}(\mathcal{B}, \mathbb{F})$, while its action on $H_{c}^{2 N}\left(\mathbb{A}^{N}, \mathbb{F}\right)$ is multiplication by $(-1)^{N}$ (the $N^{\text {th }}$ Tate twist of the trivial module). The result is now clear.

Combining the last two results, we obtain

Corollary 5.9. (cf. Theorem 1.8 above.) Maintaining the above notation, let $\xi \in$ $\mathfrak{G}(\mathbb{R})$. Then, writing $N(\xi)$ for the number of positive roots of $L:=Z_{G}(\xi)^{0}$, we have

$$
\Lambda_{\mathrm{c}}\left(\sigma, \mathcal{T}^{\xi}(G), \mathcal{S}_{\epsilon}(G)\right)=\epsilon_{0}(G) \epsilon_{0}(L) \epsilon\left(v_{\xi}\right)(-1)^{N(\xi)} .
$$

Note that Corollary 5.9, together with Theorem 4.5 and Lemma 5.2, complete the proof of Theorem 1.8.

\section{Fourier-Sato transform of Equivariant SheAves}

In this section we will treat the theory of Fourier-Sato transform for conical constructible sheaves on $\Gamma$-equivariant vectorbundles over topological spaces with a $\Gamma$-action. Since $\Gamma$ is discrete, this is a straightforward generalisation of the nonequivariant theory.

We begin with an exposition which follows very closely that of Kashiwara and Schapira [KS], including, largely, their notation. In the next section (7) below, we introduce the notation for Fourier-Sato transform which we shall continue with, and state its essential properties, particularly as they pertain to complex vector bundles which have a real structure, which is where we shall apply them.

6.1. Definitions. Let $X$ be a locally compact topological space with a (left) action of a discrete group $\Gamma$. For $g \in \Gamma$ we denote the corresponding automorphism of $X$ by $x \mapsto g x$.

Let $\mathbb{F}$ be a field of characteristic zero. Recall that a $\Gamma$-sheaf (or $\Gamma$-equivariant sheaf) of $\mathbb{F}$-vector spaces on $X$ is a sheaf $\mathcal{S}$ of $\mathbb{F}$-vector spaces together with a collection of isomorphisms

$$
\left\{\alpha_{g}: \mathcal{S} \stackrel{\sim}{\rightarrow} g^{*} \mathcal{S}\right\}_{g \in \Gamma}
$$

such that $\alpha_{1}$ is the identity map and $\alpha_{g h}=h^{*}\left(\alpha_{g}\right) \circ \alpha_{h}$ for all $g, h \in \Gamma$. With the obvious notion of equivariant morphisms, this gives us an abelian category with enough injectives. We denote the corresponding derived category of bounded complexes of $\Gamma$-equivariant sheaves by

$$
\mathcal{D}^{b}(X ; \Gamma, \mathbb{F})
$$

When $f: X \rightarrow Y$ is a continuous equivariant map such that $f_{*}$ and $f_{\text {! }}$ have finite cohomological dimension, then we have the usual functors $R f_{*}, R f_{!}, f^{*}, f^{!}$between $\mathcal{D}^{b}(X ; \Gamma, \mathbb{F})$ and $\mathcal{D}^{b}(Y ; \Gamma, \mathbb{F})$ and we also have the internal derived functors $R \mathcal{H o m}$ and $\otimes^{L}$. For the inclusion of a locally closed subspace $i: V \hookrightarrow X$ we will often write

$$
\begin{aligned}
& (-)_{V}:=R i_{i} i^{*} \\
& (-)_{V}^{\prime}:=R i_{*} i !
\end{aligned}
$$


We say that a $\Gamma$-sheaf of $\mathbb{F}$-vector spaces $\mathcal{S}$ on $X$ is constant if $\mathcal{S}$ is the pullback of a representation $V$ of $\Gamma$ under the constant mapping $X \rightarrow$ pt. We will then write $\mathcal{S}=V_{X}$. In particular we have the trivial constant $\Gamma$-sheaf $\mathbb{F}_{X}$ associated to the trivial representation $\mathbb{F}$.

6.1.1. Conical sheaves. Let $\pi: E \rightarrow X$ be a $\Gamma$-equivariant real vector bundle (i.e., $E$ is a real vector bundle on $X$, with an action of $\Gamma$ on $E$ compatible with the action on $X$ and linear on the fibres). Fibre-wise multiplication gives an action of the multiplicative group of strictly positive real numbers $\mathbb{R}^{>0}$ on $X$ which commutes with the action of $\Gamma$. We say that a $\Gamma$-sheaf $\mathcal{S}$ of $\mathbb{F}$-vector spaces on the vector bundle $E$ is conical if $\mathcal{S}$ is locally constant (hence constant) on each $\mathbb{R}^{>0}$-orbit. Denote by $\mathcal{D}_{\mathbb{R}>0}^{b}(E ; \Gamma, \mathbb{F})$ the full subcategory of the derived category of bounded complexes of $\Gamma$-sheaves of $\mathbb{F}$-vector spaces on $E$ consisting of the complexes whose cohomology sheaves are conical.

6.1.2. The equivariant Fourier-Sato transform. Let

$$
\pi: E \rightarrow X
$$

be a $\Gamma$-equivariant real vector bundle. Let

$$
\check{\pi}: \check{E} \rightarrow X
$$

be the dual of $E$ with its canonical $\Gamma$-action. Let

$$
E \times{ }_{X} \check{E}
$$

be the Whitney sum, with $p_{1}, p_{2}$ the projections onto the first, resp. second factor and let

$$
\mu: E \times_{X} \check{E} \rightarrow X \times \mathbb{R}
$$

be the duality map. We write

$$
\langle-,-\rangle: E \times_{X} \check{E} \rightarrow \mathbb{R}
$$

for $\mu$ followed by the projection onto the second factor. Then $\langle\zeta, \xi\rangle=\langle g \cdot \zeta, g \cdot \xi\rangle$, so the subspaces

$$
\begin{aligned}
& P^{\geq 0}:=\left\{(x, y) \in E \times_{X} \check{E} \mid\langle x, y\rangle \geq 0\right\} \\
& P^{\leq 0}:=\left\{(x, y) \in E \times_{X} \check{E} \mid\langle x, y\rangle \leq 0\right\}
\end{aligned}
$$

are $\Gamma$-equivariant.

Define:

$$
\begin{array}{lll}
\Psi_{P \geq_{0}}:=R p_{2_{*}} \circ R(-)_{P \geq 0}^{\prime} \circ p_{1}^{*} & : \mathcal{D}_{\mathbb{R}>0}^{b}(E ; \Gamma, \mathbb{F}) \rightarrow \mathcal{D}_{\mathbb{R}>0}^{b}(\check{E} ; \Gamma, \mathbb{F}) \\
\Phi_{P \leq 0}:=R p_{2 !} \circ(-)_{P \leq 0} \circ p_{1}^{*} & : \mathcal{D}_{\mathbb{R}>0}^{b}(E ; \Gamma, \mathbb{F}) \rightarrow \mathcal{D}_{\mathbb{R}>0}^{b}(\check{E} ; \Gamma, \mathbb{F}) \\
\Psi_{P \leq 0}:=R p_{1 *} \circ R(-)_{P \leq 0}^{\prime} \circ p_{2}^{!} & : \mathcal{D}_{\mathbb{R}>0}^{b}(\check{E} ; \Gamma, \mathbb{F}) \rightarrow \mathcal{D}_{\mathbb{R}>0}^{b}(E ; \Gamma, \mathbb{F}) \\
\Phi_{P \geq_{0}}:=R p_{1 !} \circ(-)_{P \geq 0} \circ p_{2}^{!} & : \mathcal{D}_{\mathbb{R}>0}^{b}(\check{E} ; \Gamma, \mathbb{F}) \rightarrow \mathcal{D}_{\mathbb{R}>0}^{b}(E ; \Gamma, \mathbb{F})
\end{array}
$$

It follows from the definitions that $\Phi_{P \leq 0}$ is left adjoint to $\Psi_{P \geq_{0}}$.

The following statement is much less formal (and probably the key to many of the deeper properties of the Fourier-Sato transform). 
Theorem 6.1. [KS, Theorem 3.7.7] There are natural isomorphisms of functors $\Psi_{P \geq_{0}} \simeq \Phi_{P \leq 0}$ and $\Psi_{P \leq_{0}} \simeq \Phi_{P \geq_{0}}$.

Proof. It is easy to check that all the isomorphisms in the proof of [KS, Theorem 3.7.7] are equivariant.

We now define the Fourier-Sato transform

$$
(-)^{\wedge}:=\Psi_{P \geq 0}=\Phi_{P \leq 0}: \mathcal{D}_{\mathbb{R}>0}^{b}(E ; \Gamma, \mathbb{F}) \rightarrow \mathcal{D}_{\mathbb{R}>0}^{b}(\check{E} ; \Gamma, \mathbb{F}),
$$

and the inverse Fourier-Sato transform

$$
(-)^{\vee}:=\Psi_{P \leq 0}=\Phi_{P \geq_{0}}: \mathcal{D}_{\mathbb{R}>0}^{b}(\check{E} ; \Gamma, \mathbb{F}) \rightarrow \mathcal{D}_{\mathbb{R}>0}^{b}(E ; \Gamma, \mathbb{F}) .
$$

It is clear from the definitions that both functors are functors of triangulated categories (i.e., they respect distinguished triangles).

Using the canonical isomophism $E \simeq \check{E}$, We also get functors

$$
(-)^{\vee}: \mathcal{D}_{\mathbb{R}>0}^{b}(E ; \Gamma, \mathbb{F}) \rightarrow \mathcal{D}_{\mathbb{R}>0}^{b}(\check{E} ; \Gamma, \mathbb{F}),
$$

and

$$
(-)^{\wedge}: \mathcal{D}_{\mathbb{R}>0}^{b}(\check{E} ; \Gamma, \mathbb{F}) \rightarrow \mathcal{D}_{\mathbb{R}>0}^{b}(E ; \Gamma, \mathbb{F}) .
$$

It is easy to see that we have the relation [KS, equation 3.7.9]

$$
\mathcal{S}^{\vee} \simeq \operatorname{inv}^{*}\left(\mathcal{S}^{\wedge}\right) \otimes^{L} \check{\pi}^{!} \mathbb{F}_{X}
$$

where inv denotes multiplication by -1 on the vector bundle.

6.2. Basic properties. The equivariant Fourier-Sato transform has all the basic properties of the non-equivariant version. Below we list the most important ones. Proofs in the non-equivariant context may be found in [KS]; it is easy to verify that all the constructions and morphisms that are used in loc. cit. carry over to the equivariant situation.

The main result in the theory of the Fourier-Sato transform is the result below. The proof is not purely formal. Formal methods reduce the proof to the case of $\mathbb{F}_{U}$ for a convex open cone $U$.

Theorem 6.5. [KS, Theorem 3.7.9] Let $E \rightarrow X$ be a $\Gamma$-equivariant vector bundle over a locally compact $\Gamma$-space. The Fourier-Sato transform ${ }^{\wedge}$ and the inverse transform $\checkmark$ are mutually inverse equivalences between the triangulated categories $\mathcal{D}_{\mathbb{R}>0}^{b}(E ; \Gamma, \mathbb{F})$ and $\mathcal{D}_{\mathbb{R}>0}^{b}(\check{E} ; \Gamma, \mathbb{F})$.

The following three results are purely formal consequences of the definitions, standard properties of the functors involved, and the two main Theorems above.

For a locally compact $\Gamma$-space $V$ of finite cohomological dimension we denote by $\mathrm{D}_{V}$ the Verdier dualising complex on $V$ and let $\mathrm{D}: \mathcal{D}^{b}(V ; \Gamma, \mathbb{F}) \rightarrow \mathcal{D}^{b}(V ; \Gamma, \mathbb{F})$ be the Verdier dualising functor $R \mathcal{H}$ om $\left(-, \mathrm{D}_{V}\right)$. Since the cohomology sheaves of $\mathrm{D}_{V}$ are conical, the functor $\mathrm{D}$ sends ( $\Gamma$-equivariant) complexes with conical cohomology to ( $\Gamma$-equivariant) complexes with conical cohomology. 
Proposition 6.6. [KS, Proposition 3.7.12.iv] Let $E \rightarrow X$ be a $\Gamma$-equivariant vector bundle over a locally compact $\Gamma$-space of finite cohomological dimension. We have an isomorphism of functors

$$
\vee \circ \mathrm{D}=\mathrm{D} \circ \wedge \text { : } \mathcal{D}_{\mathbb{R}>0}^{b}(E ; \Gamma, \mathbb{F}) \rightarrow \mathcal{D}_{\mathbb{R}>0}^{b}(\check{E} ; \Gamma, \mathbb{F}) .
$$

Proposition 6.7. [KS, Proposition 3.7.14] For any equivariant morphism $f: E_{1} \rightarrow$ $E_{2}$ of $\Gamma$-equivariant vector bundles over $X$ with dual morphism ${ }^{t} f: \check{E}_{2} \rightarrow \check{E}_{1}$ we have functorial isomorphisms

$$
\begin{aligned}
{ }^{t} f^{*}\left(\mathcal{S}_{1}^{\wedge}\right) & \simeq\left(R f_{!} \mathcal{S}_{1}\right)^{\wedge}, \\
{ }^{t} f^{!}\left(\mathcal{S}_{1}^{\wedge}\right) & \simeq\left(R f_{*} \mathcal{S}_{1}\right)^{\wedge} \otimes{ }^{t} f^{!} \mathbb{F}_{\check{E}_{1}}, \\
\left(f^{!} \mathcal{S}_{2}\right)^{\wedge} & \simeq R^{t} f_{*}\left(\mathcal{S}_{2}^{\wedge}\right), \\
\left(f^{!} \mathbb{F}_{E_{2}} \otimes_{L} f^{*} \mathcal{S}_{2}\right)^{\wedge} & \simeq R^{t} f_{!}\left(\mathcal{S}_{2}^{\wedge}\right),
\end{aligned}
$$

for $\mathcal{S}_{i} \in \mathcal{D}_{\mathbb{R}>0}^{b}\left(E_{i} ; \Gamma, \mathbb{F}\right)$. Analogous formulae hold when $\wedge$, * and ! are respectively replaced by $\vee$, ! and $*$ above.

The next result states that the Fourier-Sato transform is essentially independent of the base.

Proposition 6.8. [KS, Proposition 3.7.13] Let $f: Y \rightarrow X$ be an equivariant continuous map of locally compact $\Gamma$-spaces such that $f_{!}$and $f_{*}$ have finite cohomological dimension. Let $E_{X}$ be a $\Gamma$-equivariant vector bundle on $X$, and let $E_{Y}=Y \times_{X} E_{X}$ be the pull-back. Let $p_{E}: E_{Y} \rightarrow E_{X}$ and $p_{\check{E}}: \check{E}_{Y} \rightarrow \check{E}_{X}$ be the canonical projections. We have canonical isomorphisms of functors

$$
\begin{array}{lll}
\wedge \circ R\left(p_{E}\right)_{*} \simeq R\left(p_{\check{E}}\right)_{*} \circ^{\wedge} & : \mathcal{D}_{\mathbb{R}>0}^{b}\left(E_{Y} ; \Gamma, \mathbb{F}\right) \rightarrow \mathcal{D}_{\mathbb{R}>0}^{b}\left(\check{E}_{X} ; \Gamma, \mathbb{F}\right) \\
\wedge \circ R\left(p_{E}\right)_{!} \simeq R\left(p_{\check{E}}\right)_{!} \circ^{\wedge} & : \mathcal{D}_{\mathbb{R}>0}^{b}\left(E_{Y} ; \Gamma, \mathbb{F}\right) \rightarrow \mathcal{D}_{\mathbb{R}>0}^{b}\left(\check{E}_{X} ; \Gamma, \mathbb{F}\right) \\
\wedge \circ R\left(p_{E}\right)^{*} \simeq R\left(p_{\check{E}}\right)^{*} \circ^{\wedge} & : & \mathcal{D}_{\mathbb{R}>0}^{b}\left(E_{X} ; \Gamma, \mathbb{F}\right) \rightarrow \mathcal{D}_{\mathbb{R}>0}^{b}\left(\check{E}_{Y} ; \Gamma, \mathbb{F}\right) \\
\wedge \circ R\left(p_{E}\right)^{!} \simeq R\left(p_{\check{E}}\right)^{!} \circ^{\wedge} & : & : \mathcal{D}_{\mathbb{R}>0}^{b}\left(E_{X} ; \Gamma, \mathbb{F}\right) \rightarrow \mathcal{D}_{\mathbb{R}>0}^{b}\left(\check{E}_{Y} ; \Gamma, \mathbb{F}\right) .
\end{array}
$$

\section{Fourier-Sato transforms of SheAves on COMPlex VeCtor BundLES WITH REAL STRUCTURE}

We shall apply the above constructions when $E$ and $X$ are complex algebraic varieties defined over $\mathbb{R}$, with $\Gamma=\operatorname{Gal}(\mathbb{C} / \mathbb{R})$ acting via the antiholomorphic involution $\sigma$. Specifically, we shall restrict attention to a certain subcategory of $D_{\mathbb{R}>0}^{b}(E(\mathbb{C}) ; \Gamma, \mathbb{F})$ (which in this setting is denoted $D_{\mathbb{R}>0}^{b}(E(\mathbb{C}) ; \sigma, \mathbb{F})$ ) to be defined below.

Let $V$ be a semi-algebraic space with an involution $\sigma$. Recall that a sheaf $\mathcal{S}$ on a semi-algebraic variety $V$ is called semi-algebraically constructible if $V$ admits a finite semi-algebraic stratification such that $\mathcal{S}$ is locally constant on each stratum. If $\mathcal{S}$ is $\sigma$-equivariant, it is not hard to see that this stratification can be refined to a $\sigma$-equivariant stratification, with each stratum either in $V^{\sigma}$ or in $V-V^{\sigma}$. 
Let $X$ be a complex algebraic variety defined over $\mathbb{R}$ and let $E \rightarrow X$ be a complex algebraic vector bundle defined over $\mathbb{R}$. As above, fix a coefficient field $\mathbb{F}$ (of characteristic zero) for our sheaves. As pointed out in the proof of Theorem 4.5, the sets of complex points $X(\mathbb{C})$ and $E(\mathbb{C})$ have a canonical semi-algebraic structure for which complex conjugation is a semi-algebraic morphism.

Let $D_{c c}^{b}(E(\mathbb{C}) ; \sigma, \mathbb{F})$ be the full subcategory of $D_{\mathbb{R}>0}^{b}(E(\mathbb{C}) ; \sigma, \mathbb{F})$ consisting of the complexes of which the cohomology sheaves are semi-algebraically constructible with respect to some $\mathbb{R}^{>0}$-equivariant semi-algebraic stratification of $E$. We will refer to the objects of $D_{c c}^{b}(E ; \sigma, \mathbb{F})$ as complexes with conical constructible cohomology.

It is easy to check from the definitions that the Fourier-Sato transform

$$
\mathcal{F}: D_{\mathbb{R}>0}^{b}(E(\mathbb{C}) ; \sigma, \mathbb{F}) \rightarrow D_{\mathbb{R}>0}^{b}(\check{E}(\mathbb{C}) ; \sigma, \mathbb{F})
$$

sends complexes with constructible cohomology to complexes with constructible cohomology. Hence it induces a Fourier-Sato transform

$$
\mathcal{F}: D_{c c}^{b}(E(\mathbb{C}) ; \sigma, \mathbb{F}) \rightarrow D_{c c}^{b}(\check{E}(\mathbb{C}) ; \sigma, \mathbb{F})
$$

We shall deal with bundles $E \longrightarrow X$ as above which are $\Gamma$ (or $\sigma$ )-equivariant; then $\sigma$ acts on the cohomology sheaves, which are equivariant in the sense of the above discussion. In this section we shall first reformulate the basic properties of the Fourier-Sato transform functor for this context, and since we shall be interested in the stalks of the cohomology sheaves of Fourier-Sato transforms and their connection with Euler characteristics, we discuss below certain constructible functions associated with objects in $\mathcal{D}_{c c}^{b}(E ; \sigma, \mathbb{F})$.

7.1. Basic properties of the Fourier-Sato transform. To formulate the basic properties of the Fourier-Sato transform efficiently, we shall require the functors $\mathfrak{t}$ (Tate twist) and $\mathfrak{s h}$ (shift) which take a complex $\mathcal{S} \in \mathcal{D}_{c c}^{b}(E ; \sigma, \mathbb{F})$ to $\mathcal{S}(1)$ and $\mathcal{S}[1]$ respectively, where the former is $\mathcal{S}$, with $\sigma$ action twisted by the sign representation, and the latter is $\mathcal{S}$, shifted one degree to the left.

The Fourier-Sato transform in this special setting will be denoted $\mathcal{F}=$ $\mathcal{F}_{E}: \mathcal{D}_{c c}^{b}(E ; \sigma, \mathbb{F}) \longrightarrow \mathcal{D}_{c c}^{b}(\check{E} ; \sigma, \mathbb{F})$, where $(-)^{\wedge}=\mathcal{F}_{E}(-)$ (see 6.2$)$. We also write $\mathcal{F}_{E}^{\prime}: \mathcal{D}_{c c}^{b}(E ; \sigma, \mathbb{F}) \longrightarrow \mathcal{D}_{c c}^{b}(\check{E} ; \sigma, \mathbb{F})$ for the functor defined by $\mathcal{F}_{E}^{\prime}(-)=(-)^{\vee}$. Note that both $\mathcal{F}_{E}$ and $\mathcal{F}_{E^{\prime}}$ commute with $\mathfrak{t t}$ and $\mathfrak{s h}$.

We will repeatedly use the following observation.

Lemma 7.1. Let $X$ be an algebraic variety defined over $\mathbb{R}$. Let $\pi: E \rightarrow X$ be a complex algebraic vector bundle of rank $r$ defined over $\mathbb{R}$. Then

$$
\begin{aligned}
\pi ! \mathbb{F}_{E} & =\mathbb{F}_{X}(-r)[-2 r] \\
\pi^{!} \mathbb{F}_{X} & =\mathbb{F}_{E}(r)[2 r] .
\end{aligned}
$$

Proof. The first formula follows from the fact that for the real vector bundle $\mathbb{A}^{r}:=\mathbb{C}^{r}$ with $\Gamma$ acting via complex conjugation, we have that $H_{c}^{i}\left(\mathbb{A}^{r}, \mathbb{F}\right)$ is isomorphic to $\mathbb{F}(-r)$ when $i=2 r$ and 0 otherwise. The second formula follows from Verdier duality. 
We shall now reformulate the basic properties of the Fourier-Sato transform for this context. First observe that (6.4) may be written as

$$
\begin{aligned}
\mathcal{S}^{\vee} \simeq \operatorname{inv}^{*}\left(\mathcal{S}^{\wedge}\right) \otimes^{L} \check{\pi}^{!} \mathbb{F}_{X} & \simeq \operatorname{inv}^{*}\left(\mathcal{S}^{\wedge}\right)(\operatorname{rank} E)[2 \operatorname{rank} E] \\
& \simeq \mathfrak{t t}^{\operatorname{rank} E_{\mathfrak{s h}}}{ }^{2 \operatorname{rank} E_{\operatorname{inv}^{*}}\left(\mathcal{S}^{\wedge}\right),}
\end{aligned}
$$

where $\operatorname{rank} E$ denotes the rank of $E$ as a complex vector bundle. It follows that

$$
\mathcal{F}_{E}^{\prime} \simeq \mathfrak{t t}^{\text {rank } E} \circ \mathfrak{s h}^{2 \operatorname{rank} E} \circ \text { inv }^{*} \circ \mathcal{F}_{E} .
$$

Writing (6.5) in the present notation, we have

$$
\mathcal{F}_{\check{E}} \circ \mathcal{F}_{E}^{\prime}=\operatorname{id}_{\mathcal{D}_{c c}^{b}(E ; \sigma, \mathbb{F})} .
$$

It follows that

$$
\mathcal{F}_{\check{E}} \circ \mathcal{F}_{E}=\mathfrak{t t}^{-\operatorname{rank} E} \circ \mathfrak{s h}^{-2 \operatorname{rank} E} \circ \mathrm{inv}^{*} .
$$

The Verdier dualising functor preserves the category $\mathcal{D}_{c c}^{b}(E ; \sigma, \mathbb{F})$, and hence induces $D: \mathcal{D}_{c c}^{b}(E ; \sigma, \mathbb{F}) \longrightarrow \mathcal{D}_{c c}^{b}(E ; \sigma, \mathbb{F})$. It follows from (6.4) and (6.6) that, if $E \longrightarrow X$ above is a complex vector bundle with $\Gamma$-equivariant real structure over a $\Gamma$-space of finite cohomological dimension, then

$$
D \circ \mathcal{F}_{E}=\mathfrak{t t}^{\operatorname{rank} E} \circ \mathfrak{s h}^{2 \operatorname{rank} E} \circ \operatorname{inv}^{*} \circ \mathcal{F}_{E} \circ D .
$$

To describe the behaviour of Fourier-Sato transform under maps of equivariant complex bundles, suppose that $f: E_{1} \longrightarrow E_{2}$ is a morphism of $\Gamma$-equivariant complex vector bundles over $X$, and let $\check{f}: \check{E}_{2} \longrightarrow \check{E}_{1}$ be the dual morphism. Then the first line of the equation in Proposition 6.7 may be written

$$
\check{f}^{*} \circ \mathcal{F}_{E_{1}}=\mathcal{F}_{E_{2}} \circ R f_{!} \text {. }
$$

Now suppose that $\mathcal{L}$ is a local system on $X$, that $\pi: E \longrightarrow X$ is a vector bundle of rank $r$ satisfying the conditions of the first two paragraphs of this section, and let $i_{0}: X \longrightarrow \check{E}$ be the zero section. Then the fourth line of (6.7) becomes

$$
\mathcal{F}_{E} \pi^{*} \mathcal{L}=\mathfrak{s h}^{-2 r} \mathfrak{t t}^{-r} i_{0 !} \mathcal{L}
$$

We next describe the effect of base change on Fourier-Sato transform. Let $f: Y \longrightarrow X$ be a morphism of complex algebraic varieties defined over $\mathbb{R}$, let $E_{X} \rightarrow X$ be a complex vector bundle defined over $\mathbb{R}$, and let $E_{Y}:=Y \times_{X} E_{X}$ be the pull-back of $E_{X}$. We have maps as in the diagrams (7.9) below.

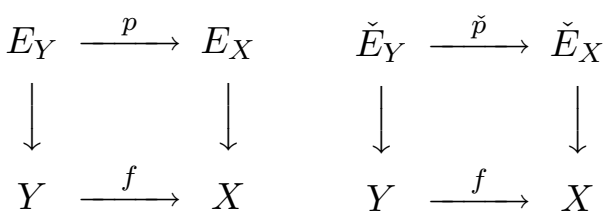

Then we have from Proposition 6.8,

$$
\mathcal{F}_{E_{X}} \circ R p_{!}=R \check{p}_{!} \circ \mathcal{F}_{E_{Y}} .
$$

Finally, we shall require the observation that a shift of the Fourier-Sato transform above preserves perversity. 
Proposition 7.11. (cf. [Sho, 16.5]) The functor $\mathfrak{s h}^{\text {rank } E} \circ \mathcal{F}_{E}$ takes perverse sheaves in $\mathcal{D}_{c c}^{b}(E ; \sigma, \mathbb{F})$ to perverse sheaves in $\mathcal{D}_{c c}^{b}(\check{E}) ; \sigma, \mathbb{F}$.

The reason why the shift is necessary in (7.11) may be seen by comparing [Sho, 16.3.2] and our (7.7) above.

7.2. Geometric Fourier-Sato transform and constructible functions. Let $R(\sigma)$ be the $\mathbb{F}$-representation ring of $\Gamma:=\langle\sigma\rangle$ and let $\mathcal{F}_{c c}(E(\mathbb{C}) / \sigma, R(\sigma))$ be the group of $R(\sigma)$-valued functions on the orbit space $E(\mathbb{C}) / \sigma$ which are $\mathbb{R}^{>0}$-invariant, semi-algebraically constructible, and whose value at the orbit of $x \notin E(\mathbb{R})$ is a multiple of the regular representation of $\langle\sigma\rangle$. For any complex $K^{\bullet}$ in $D_{c c}^{b}(E(\mathbb{C}) ; \sigma, \mathbb{F})$ we define the orbit characteristic function $\chi_{K} \bullet \in \mathcal{F}_{c c}(E(\mathbb{C}) / \sigma, R(\sigma))$ of $K^{\bullet}$ to be the function whose value at the orbit $\bar{x}$ of a point $x \in E(\mathbb{C}) / \sigma$ is the element of $R(\sigma)$ given by

$$
\chi_{K} \bullet(\bar{x})=\bigoplus_{y \in\{x, \sigma(x)\}} \sum_{i}(-1)^{i}\left[\mathcal{H}^{i}\left(K^{\bullet}\right)_{y}\right] .
$$

Note that if $x \notin E(\mathbb{R})$, the $\sigma$-equivariance of $K^{\bullet}$ implies that $\chi_{K} \bullet(\bar{x})$ is induced from the trivial subgroup of $\langle\sigma\rangle$, and hence is a multiple of the regular representation of $\langle\sigma\rangle$.

Our strategy will be to identify the weighted Euler characteristics in which we are interested with "characteristic functions" of $\sigma$-equivariant complexes of sheaves, and apply the Fourier-Sato transform to obtain our main result. For the following definition, observe that for any $\Gamma$-equivariant sheaf $\mathcal{S}$ on a $\Gamma$-space $X$ we have an induced isomorphism $\mathcal{S}_{x} \stackrel{\sim}{\rightarrow} \mathcal{S}_{\sigma(x)}$ of stalks at $x, \sigma(x) \in X$. In particular, we have a $\Gamma$-action on the stalks $\mathcal{S}_{x}$ at the fixed points $x \in X^{\sigma}$.

Definition 7.13. Let $K^{\bullet} \in \mathcal{D}_{c c}^{b}(X ; \sigma, \mathbb{F})$, where $X$ is a complex algebraic variety which is defined over $\mathbb{R}$. Define the characteristic function $\Lambda_{K} \bullet: X^{\sigma}=X(\mathbb{R}) \longrightarrow \mathbb{F}$ by

$$
\Lambda_{K} \bullet(x)=\sum_{i}(-1)^{i} \operatorname{Trace}\left(\sigma, \mathcal{H}^{i}\left(K^{\bullet}\right)_{x}\right) .
$$

We shall require the following key result.

Proposition 7.15. Let $X$ be a complex algebraic variety defined over $\mathbb{R}$ and let $E \rightarrow$ $X$ be a complex algebraic vector bundle defined over $\mathbb{R}$. Let $M^{\bullet}, N^{\bullet}$ be two complexes of sheaves in $D_{c c}^{b}(E(\mathbb{C}) ; \sigma, \mathbb{F})$ which have equal orbit characteristic functions.

Then $\mathcal{F}\left(M^{\bullet}\right)$ and $\mathcal{F}\left(N^{\bullet}\right)$ have equal orbit characteristic functions $\chi_{\mathcal{F}(M \bullet)}: \check{E}(\mathbb{C}) / \sigma \rightarrow R(\sigma)$.

Proof. We are given that

$$
\chi_{M} \bullet=\chi_{N} \bullet
$$

Let $K_{c c}(E(\mathbb{C}) ; \sigma, \mathbb{F})$ be the Grothendieck group of the triangulated category $D_{c c}^{b}(E(\mathbb{C}) ; \sigma, \mathbb{F})$. The orbit characteristic function map induces a homomorphism

$$
\chi: K_{c c}(E(\mathbb{C}) ; \sigma, \mathbb{F}) \rightarrow \mathcal{F}_{c c}(E(\mathbb{C}) / \sigma, R(\sigma)) .
$$

Since the semi-algebraic topology is sufficiently fine, this homomorphism is an isomorphism. (This is the equivariant analogue of [KS, Th. 9.7.1] using semi-algebraic 
rather than subanalytic constructibility.) Indeed, surjectivity is easy to check, and the injectivity follows from the fact that semi-algebraic triangulation gives us for any complex $K^{\bullet}$ in $D_{c c}^{b}(E(\mathbb{C}) ; \sigma, \mathbb{F})$ a (finite) $\mathbb{R}^{>0}$-equivariant semi-algebraic stratification of $E(\mathbb{C})$, with each stratum either contained in $E(\mathbb{R})$ or equal to a disjoint union of the form $U \coprod \sigma \cdot U$, such that every cohomology sheaf of $K^{\bullet}$ is constant on each stratum.

By the injectivity of $\chi$, our hypotheses imply that

$$
\left[M^{\bullet}\right]=\left[N^{\bullet}\right] \in K_{c c}(E(\mathbb{C}) ; \sigma, \mathbb{F}) .
$$

Since $\mathcal{F}$ is a functor of triangulated categories, $\mathcal{F}$ descends to a homorphism of Grothendieck groups. In particular, this implies that

$$
\left[\mathcal{F}\left(M^{\bullet}\right)\right]=\left[\mathcal{F}\left(N^{\bullet}\right)\right] \in K_{c c}(\check{E}(\mathbb{C}) ; \sigma, \mathbb{F}) .
$$

Applying $\chi$, it follows that $\mathcal{F}\left(M^{\bullet}\right)$ and $\mathcal{F}\left(N^{\bullet}\right)$ have the same orbit characteristic function $\check{E}(\mathbb{R}) \rightarrow R(\sigma)$.

For practical application of Proposition 7.15 it is useful to note

Lemma 7.16. With notation as in Proposition 7.15 , let $M^{\bullet}$ and $N^{\bullet}$ be complexes in $D_{c c}^{b}(E(\mathbb{C}) ; \sigma, \mathbb{F})$ which satisfy

(i) For every $x \in E(\mathbb{C})-E(\mathbb{R})$, the stalks of $M^{\bullet}$ and $N^{\bullet}$ have equal EulerPoincaré characteristics:

$$
\sum_{i \in \mathbb{Z}}(-1)^{i} \operatorname{dim}_{\mathbb{F}} \mathcal{H}^{i}\left(M^{\bullet}\right)_{x}=\sum_{i \in \mathbb{Z}}(-1)^{i} \operatorname{dim}_{\mathbb{F}} \mathcal{H}^{i}\left(N^{\bullet}\right)_{x} .
$$

(ii) For every $x \in E(\mathbb{R})$, the virtual representations of $\Gamma$ on the stalks at $x$ of $M$ and $N$ are equal in the Grothendieck group:

$$
\sum_{i \in \mathbb{Z}}(-1)^{i}\left[\mathcal{H}^{i}\left(M^{\bullet}\right)_{x}\right]=\sum_{i \in \mathbb{Z}}(-1)^{i}\left[\mathcal{H}^{i}\left(N^{\bullet}\right)_{x}\right] .
$$

Then $M^{\bullet}$ and $N^{\bullet}$ have equal orbit characteristic functions and hence the conclusion of Proposition 7.15 holds.

Proof. For $x \in E(\mathbb{C})-E(\mathbb{R})$, both $\chi_{M} \bullet(x)$ and $\chi_{N} \bullet(x)$ are multiples of the regular representation of $\Gamma$, and (i) implies that they are equal. If $x \in E(\mathbb{R})$, (ii) states that $\chi_{M} \cdot(x)=\chi_{N} \cdot(x)$.

The next result, which will be the principal application of these considerations, follows immediately.

Corollary 7.17. With notation as in Proposition 7.15, if $M^{\bullet}$ and $N^{\bullet}$ are complexes in $D_{c c}^{b}(E(\mathbb{C}) ; \sigma, \mathbb{F})$ which satisfy the hypotheses (i) and (ii) of Lemma 7.16, then the characteristic functions $\Lambda_{\mathcal{F} M} \bullet$ and $\Lambda_{\mathcal{F} N} \bullet: \check{E}(\mathbb{R}) \longrightarrow \mathbb{Z}$ are equal.

\section{VARIETIES OF tORI AND Springer Representations}

In this section, we discuss situations in which we shall apply (7.13) and (7.17). To simplify the discussion we take our coefficient field $\mathbb{F}$ (of characteristic zero) to be algebraically closed (although this is not needed to define the local system $\mathcal{S}_{\epsilon}$ or to prove the main results). 
Let $G$ be a connected reductive $\mathbb{R}$-group, and as in $\S 1$, write $\mathfrak{G}$ for the Lie algebra, $\mathcal{T}$ for the variety of tori, $\mathcal{P}$ for the variety of Killing pairs, and $\mathcal{B}$ for the variety of Borel subgroups. We write $W$ for the absolute Weyl group, i.e., the Galois group of the covering $\mathcal{P} \rightarrow \mathcal{T}$, and $\widetilde{W}$ for the corresponding extension of $\Gamma$ by $W$ as in (3.2).

Let $\tilde{V}, V$ be the varieties

$$
\begin{aligned}
\tilde{V} & =\{((T \subseteq B), \xi) \mid \xi \in \operatorname{Lie} T\} \subset \mathcal{P} \times \mathfrak{G}, \\
V & =\{(T, \xi) \mid \xi \in \operatorname{Lie} T\} \subset \mathcal{T} \times \mathfrak{G} .
\end{aligned}
$$

Clearly the obvious map $\omega: \tilde{V} \longrightarrow V$ is a Galois $W$-covering of $V$ defined over $\mathbb{R}$, and we have a commutative square

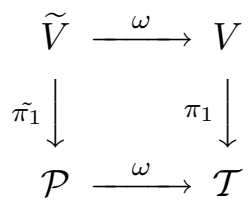

where the horizontal arrows are $W$-coverings, and the vertical maps are fibrations with fibre $\mathbb{A}^{r}$, where $r=\operatorname{dim} T_{0}$ is the rank of $G$ (as complex algebraic group). We therefore have irreducible local systems $\mathcal{S}_{E}^{V}$ on $V$ for each irreducible representation $E$ of $W$, which are the pullbacks of the corresponding local systems on $\mathcal{T}$. In analogy with (1.3), if $\mathbb{F}$ denotes the constant sheaf on $\tilde{V}$, we have

$$
\omega_{!} \mathbb{F} \cong \oplus_{E \in \hat{W}} E \otimes \mathcal{S}_{E}^{V} .
$$

In general $\Gamma$ permutes the summands of $(8.3)$, and $\mathcal{S}_{E}^{V}$ is in $D_{c c}^{b}(V ; \sigma, \mathbb{F})$ precisely when the equivalence class of $E$ is fixed by $\Gamma$. As remarked in $\S 1$, this is the case when $E=\epsilon$, the sign representation of $W$. Thus $\mathcal{S}_{\epsilon}^{V}$ is $\Gamma$-equivariant.

Now let $\rho: V \longrightarrow \mathfrak{G}$ be the first projection; of course $\rho$ is not proper.

Lemma 8.4. Let $K^{\bullet}=\rho_{!} \mathcal{S}_{\epsilon}^{V}$ in $\mathcal{D}_{c c}^{b}(\mathfrak{G} ; \sigma, \mathbb{F})$, where $\epsilon$ is the sign representation of $W$. Then

(i) The orbit characteristic function $\chi_{K} \bullet($ see 7.12) is supported on the $\Gamma$-orbits in the variety of semisimple elements of $\mathfrak{G}$, and its values are given by

$$
\chi_{K} \cdot(\bar{\xi})=\sum_{i \in \mathbb{Z}}(-1)^{i} \bigoplus_{\alpha \in\{\xi, \sigma(\xi)\}}\left[H_{c}^{i}\left(\mathcal{T}^{\alpha}, \mathcal{S}_{\epsilon}\right)\right],
$$

for $\xi \in \mathfrak{G}$, where $\mathcal{S}_{\epsilon}$ is the local system on $\mathcal{T}$ defined in (1.4).

(ii) For $\xi \notin \mathfrak{G}(\mathbb{R})$, the Euler-Poincaré characteristic is given by $\sum_{i \in \mathbb{Z}}(-1)^{i} \operatorname{dim}_{\mathbb{F}} \mathcal{H}^{i}\left(K^{\bullet}\right)_{\xi}=\sum_{i \in \mathbb{Z}}(-1)^{i} \operatorname{dim} H_{c}^{i}\left(\mathcal{T}^{\xi}, \mathcal{S}_{\epsilon}\right)$.

(iii) For $\xi \in \mathfrak{G}(\mathbb{R})$ the characteristic function is given by $\Lambda_{K} \bullet(\xi)=$ $\sum_{i \in \mathbb{Z}}(-1)^{i} \operatorname{Trace}\left(\sigma, H_{c}^{i}\left(\mathcal{T}^{\xi}, \mathcal{S}_{\epsilon}\right)\right)=\Lambda_{c}\left(\sigma, \mathcal{T}^{\xi}, \mathcal{S}_{\epsilon}\right)(c f .1 .1)$.

Proof. It is clear that the stalks of $\mathcal{H}^{i}\left(K^{\bullet}\right)$ have an $\mathbb{R}^{>0}$-action under which they are invariant, so that $K^{\bullet} \in \mathcal{D}_{c c}^{b}(\mathfrak{G} ; \sigma, \mathbb{F})$.

The stalk $\mathcal{H}^{i}\left(\rho_{!} \mathcal{S}_{\epsilon}^{V}\right)_{\xi}$ at $\xi \in \mathfrak{G}$ is isomorphic to $H_{c}^{i}\left(\rho^{-1}(\xi), \mathcal{S}_{\epsilon}^{V}\right)=H_{c}^{i}\left(\mathcal{T}^{\xi}, \mathcal{S}_{\epsilon}^{V}\right)$. But the commutative square (8.2) shows that $\mathcal{S}_{\epsilon}^{V}$ coincides with the local system $\mathcal{S}_{\epsilon}$ of 1.4. The statement (i) now follows immediately from the definition (7.12). 
Bearing in mind the definitions (1.1) and (7.14), the above observations also prove (ii) and (iii).

Our next objective is to show that $K^{\bullet}$ above has, up to sign, the same characteristic function as a well-known perverse sheaf in $\mathcal{D}_{c c}^{b}(\mathfrak{G} ; \sigma, \mathbb{F})$. To set the scene, consider the following diagram:

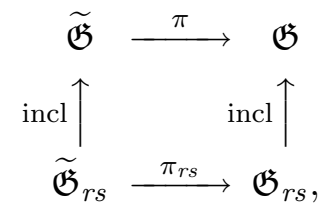

where

$$
\widetilde{\mathfrak{G}}=\{(B, \xi) \mid \xi \in \operatorname{Lie} B\} \subset \mathcal{B} \times \mathfrak{G},
$$

$\mathfrak{G}_{r s}$ is the variety of regular and semisimple elements of $\mathfrak{G}, \pi, \pi_{r s}$ are the respective second projections, and $\widetilde{\mathfrak{G}}_{r s}=\pi_{r s}^{-1}\left(\mathfrak{G}_{r s}\right)$. Note that the bottom row of $(8.5)$ is an unramified covering with group $W$. Similarly, we have that the map $\rho: V \rightarrow \mathfrak{G}$ induces an isomorphism

$$
\rho_{r s}: \rho^{-1}\left(\mathfrak{G}_{r s}\right) \stackrel{\sim}{\rightarrow} \mathfrak{G}_{r s}
$$

and the corresponding map $\widetilde{\rho}: \widetilde{V}$ to $\widetilde{G}$ (that sends a triple $(B, T, \xi)$ to the pair $(B, \xi)$ ) induces an isomorphism

$$
\widetilde{\rho}_{r s}: \rho^{-1}(\widetilde{V}) \stackrel{\sim}{\rightarrow} \mathfrak{G}_{r s} .
$$

The relationship between the various spaces and Galois covering which have occurred in this work, is summarised in the following two diagrams.

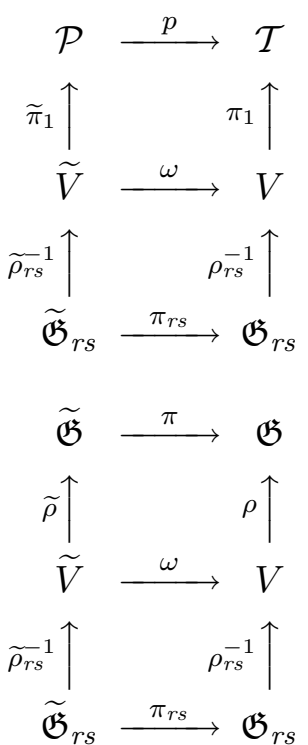

Here the maps $\pi_{r s}, \omega$ and $p$ are all Galois $W$-coverings, whereas the maps $\widetilde{\pi}_{1}$ and $\pi_{1}$ are affine fibrations. Let us stress that $\pi$ is not a Galois $W$-covering. 
Write $\mathcal{Y}$ for the $\Gamma$-equivariant local system $\left(\pi_{r s}\right)_{*} \mathbb{F}$ on $\mathfrak{G}_{r s}$, which corresponds to the regular representation of the covering group $W$. It is a result of Lusztig [Sho, 4.2, p.76] that

$$
I C(\mathfrak{G}, \mathcal{Y})[\operatorname{dim} \mathfrak{G}] \cong R \pi_{*} \mathbb{F}[\operatorname{dim} \mathfrak{G}],
$$

where $I C$ denotes the intersection complex extension [BBD]. This includes the statement that both sides are perverse. It is easy to check that both the construction of $I C(\mathfrak{G}, \mathcal{Y})$ and the proof of the isomorphism generalise to the $\Gamma$-equivariant context, i.e. that the statement (8.8) may be interpreted as an isomorphism in the $\Gamma$-equivariant category $\mathcal{D}_{c c}^{b}(\mathfrak{G} ; \sigma, \mathbb{F})$.

Since $\mathcal{Y}$ clearly has a $\Gamma$-equivariant $W$-action, so does the right side of (8.8) and hence so does the cohomology of this perverse sheaf. This is the Springer action. In particular, we obtain an action of $W$ on the stalk of $R^{i} \pi_{*} \mathbb{F}$ at each $\xi \in \mathfrak{G}(\mathbb{R})$, which is isomorphic to $H^{i}\left(\pi^{-1}(\xi), \mathbb{F}\right)=H^{i}\left(\mathcal{B}_{\xi}, \mathbb{F}\right)$, where $\mathcal{B}_{\xi}=\{B \in \mathcal{B} \mid \xi \in$ Lie $\mathcal{B}\}$.

Observe that since $\Gamma$ acts as an automorphism group of $W$, it permutes the irreducible $\mathbb{F}$-representations of $W$. Say that the irreducible representation $E$ of $W$ is $\Gamma$-invariant if each element of $\Gamma$ takes $E$ to an equivalent representation.

Definition 8.9. Write $M^{\bullet}$ for the shift $R \pi_{*} \mathbb{F}$ of the perverse sheaf in (8.8). For any irreducible $\Gamma$-invariant representation $E$ of $W$, we write $M_{E}^{\bullet}$ for the $E$-isotypic direct summand of $M^{\bullet}$ corresponding to the intersection complex extension $I C\left(\mathcal{Y}_{E}\right)$ of the ( $\Gamma$-equivariant!) direct summand $\mathcal{Y}_{E}$ of the local system $\mathcal{Y}$.

Observe that for $E$ as above, we have an isomorphism

$$
M_{E}^{\bullet} \cong R \mathcal{H o m}_{W}\left(E, M^{\bullet}\right) \text {. }
$$

of $\Gamma$-equivariant perverse sheaves (cf. [Sho, §5]).

Theorem 8.11. Let $G$ be a complex algebraic group which is defined over $\mathbb{R}$. Let $K^{\bullet}=\rho ! \mathcal{S}_{\epsilon}^{V}$ be as in Lemma 8.4 and $M_{\epsilon}^{\bullet}$ as in Definition 8.9, with $E=\epsilon$. Then $K^{\bullet}$ and $M_{\epsilon}^{\bullet}$ are objects in $\mathcal{D}_{c c}^{b}(\mathfrak{G} ; \sigma, \mathbb{F})$, and have the same orbit characteristic function: $\mathfrak{G}(\mathbb{C}) / \sigma \longrightarrow R(\sigma)$.

Before giving the proof, we need several results, some of which are $\Gamma$-equivariant versions of standard results from the literature. The latter are due to Borho-MacPherson and Lusztig, but the exposition by Shoji [Sho] is a good reference, although the treatment there is oriented towards the case of finite characteristic. The references in [op. cit.] suffice for a complete exposition of the proofs. We begin with the following observation concerning the action of $\sigma$ on the Springer representations.

Lemma 8.12. The action of $\sigma$ on $\mathcal{B}$ induces an action of $\sigma$ on $\mathcal{B}_{\xi}$ for any $\xi \in \mathfrak{G}(\mathbb{R})$. The Springer action of $W$ defined above and this action of $\sigma$ generate an action of the group $\widetilde{W}$ (as in (3.3)) on $H^{i}\left(\mathcal{B}_{\xi}, \mathbb{F}\right)$, with $\gamma$ acting via $\sigma$.

Proof. The lemma is a restatement of the fact that the action of $W$ on $H^{i}\left(\mathcal{B}_{\xi}, \mathbb{F}\right)=$ $\left(R \pi_{*} \mathbb{F}\right)_{\xi}$ is $\Gamma$-equivariant for $\xi \in \mathfrak{G}(\mathbb{R})$, which follows immediately from the fact that the Springer action of $W$ on $R \pi_{*} \mathbb{F}$ is $\Gamma$-equivariant. 
Lemma 8.13. (cf. [Sho, Prop. 5.4 and its proof]) We have for each $i \in \mathbb{Z}$, an isomorphism of $\widetilde{W}$-modules

$$
H^{i}(\mathcal{B}, \mathbb{F}) \stackrel{\cong}{\longrightarrow} \operatorname{Hom}_{\mathbb{F}}\left(H_{c}^{4 N-i}(\mathcal{P}, \mathbb{F}), \mathbb{F}\right),
$$

where the action on the left arises from the Springer action of $\widetilde{W}$ (see (8.12) above) and the action on the right arises functorially from the action of $\widetilde{W}$ on $\mathcal{P}$.

Proof. Since the map $\mathcal{P} \longrightarrow \mathcal{B}$ is a fibration with fibre $\mathbb{A}^{N}$, we have, for each integer $i$, an isomorphism of vector spaces

$$
H^{i}(\mathcal{B}, \mathbb{F}) \stackrel{\cong}{\longrightarrow} H^{i}(\mathcal{P}, \mathbb{F})
$$

Now $W$ acts on both sides, the action on the left side being the Springer action, while the action on the right side is the classical action. But by [Sho, loc. cit.], these two actions of $W$ coincide, i.e., the isomorphism (8.14) respects the $W$ action on both sides. Since the map $\mathcal{P} \longrightarrow \mathcal{B}$ is defined over $\mathbb{R}$, the isomorphism (8.14) also respects the $\Gamma$-action, whence by $(8.12)$, the isomorphism (8.14) respects the respective actions of $\widetilde{W}$.

By Poincaré duality for $\mathcal{P}$ we have a canonical $\widetilde{W}$-equivariant isomorphism

$$
H^{i}(\mathcal{P}, \mathbb{F}) \cong \operatorname{Hom}\left(H_{c}^{4 N-i}(\mathcal{P}, \mathbb{F}), H_{c}^{4 N}(\mathcal{P}, \mathbb{F})\right) .
$$

The assertion of the Lemma now follows from the fact that $\widetilde{W}$ acts trivially on $H_{c}^{4 N}(\mathcal{P}, \mathbb{F})$, since this vector space is generated by the orientation class of the complex manifold $\mathcal{P}$ and $\sigma$ preserves the orientation, since $\mathcal{P}$ has even complex dimension.

Suppose $\xi \in \mathfrak{G}$ is semisimple. Let $L=L_{\xi}=Z_{G}(\xi)^{0}$ and $\mathfrak{L}=\operatorname{Lie}(L)$, a Levi subalgebra of $\mathfrak{G}$. Let $T$ be a maximal torus of $L$. Then $T$ is also a maximal torus of $G$. If $W_{T}:=N_{G}(T) / T$ and $W_{T}(L):=N_{L}(T) / T$, then $W_{T}(L) \subset W_{T}$. Recall from $\S 3.3$ that $W_{T}$ and $W_{T}(L)$ are the respective Galois groups of the coverings $G / T \rightarrow \mathcal{T}$ and $L / T \rightarrow \mathcal{T}(L)$. Write $W$ (resp. $W(L)$ ) for the Galois group of automorphisms of the covering $p: \mathcal{P} \rightarrow \mathcal{T}$ (resp. $p_{L}: \mathcal{P}(L) \rightarrow \mathcal{T}(L)$ ).

Choose a Borel subgroup $B_{T}$ of $G$ which contains $T$. Then $B_{T}(L):=B_{T} \cap L$ is a Borel subgroup of $L$, and thus $B_{T}$ determines isomorphisms (cf. (3.20)(ii)) $G / T \stackrel{\phi_{T}}{\longrightarrow}$ $\mathcal{P}$ and $L / T \stackrel{\phi_{T}(L)}{\longrightarrow} \mathcal{P}(L)$. Using the inclusion $L / T \hookrightarrow G / T$, we identify $\mathcal{P}(L)$ as a subvariety of $\mathcal{P}$, uniquely up to transformation by an element of $W$. Moreover $B_{T}$ also determines isomorphisms $W_{T} \cong W$ and $W_{T}(L) \cong W(L)$ as in $\S 3.3(3.20)$, and hence an embedding

$$
W(L) \hookrightarrow W
$$

in which $W(L)$ is identified as the group of covering transformations in $W$ which stabilise the subvariety $\mathcal{P}(L)$. By (3.20)(iv), this embedding of groups depends on the choice of $B_{T}$, up to conjugation by an element of $W$.

If $\xi \in \mathfrak{G}(\mathbb{R})$, then $L \subset G$ is defined over $\mathbb{R}$, and $T$ may (and will) be taken to be defined over $\mathbb{R}$. Note that in the above identification of $\mathcal{P}(L)$ as a subvariety of $\mathcal{P}$, since the maps $\phi_{T}$ and $\phi_{T}(L)$ are twists, they do not necessarily respect the actions of $\sigma$ on the various spaces. In fact the involution $\sigma$ of $\mathcal{P}$ does not generally stabilise the subvariety $\mathcal{P}(L)$ identified as above. Nonetheless, $W_{T}(L)$ is a $\sigma$-stable subgroup of 
$W_{T}$, and $\mathcal{T}(L)$ and $\mathcal{P}(L)$ inherit $\sigma$-actions, as do $W(L) \subseteq W$, and $W_{T}(L) \subseteq W_{T}$. As in (3.20)(iv), the choice of $B_{T}$ determines extensions of the above group isomorphisms to

$$
\widetilde{W_{T}} \cong \widetilde{W} \text { and } \widetilde{W_{T}(L)} \cong \widetilde{W(L)},
$$

and hence an embedding

$$
\widetilde{W(L)} \hookrightarrow \widetilde{W}
$$

which extends the above embedding $W(L) \hookrightarrow W$. Again, this embedding depends on the choice of $B_{T}$ up to conjugacy by an element of $W$.

Lemma 8.16. With notation as above, we have, for every $i \in \mathbb{Z}$, an isomorphism of $W$-modules

$$
H_{c}^{i}\left(p^{-1} \mathcal{T}\left(L_{\xi}\right), \mathbb{F}\right) \cong_{W} \operatorname{Ind}_{W\left(L_{\xi}\right)}^{W} H_{c}^{i}\left(\mathcal{P}\left(L_{\xi}\right), \mathbb{F}\right) .
$$

If $\xi \in \mathfrak{G}(\mathbb{R})$, then we have, for every $i \in \mathbb{Z}$, an isomorphism of $\widetilde{W}$-modules

$$
H_{c}^{i}\left(p^{-1} \mathcal{T}\left(L_{\xi}\right), \mathbb{F}\right) \cong \widetilde{W} \operatorname{Ind}_{\widetilde{W\left(L_{\xi}\right)}}^{\widetilde{W}} H_{c}^{i}\left(\mathcal{P}\left(L_{\xi}\right), \mathbb{F}\right) .
$$

Proof. The first assertion follows from the fact that the isomorphism of complex varieties $G / T \cong \mathcal{P}$ determined by $B_{T}$ gives a $W_{T}$-equivariant isomorphism

$$
p^{-1} \mathcal{T}\left(L_{\xi}\right) \cong W_{T} \times{ }^{W_{T}(L)} L / T .
$$

Note that we may identify the set $W_{T} / W_{T}(L)$ with the set of parabolic subgroups $P$ of $G$ that contain $L$ as a Levi subgroup. For each such $P$, the variety $\left\{\left(T^{\prime}, B\right) \mid T^{\prime} \subset\right.$ $\left.L, T^{\prime} \subset B \subset P\right\}$ is isomorphic to $\mathcal{P}(L) \cong L / T$ via the map $\left(T^{\prime}, B\right) \mapsto\left(T^{\prime}, B \cap L\right)$.

Now assume $\xi \in \mathfrak{G}(\mathbb{R})$. First observe that we have a $\widetilde{W_{T}}$-equivariant isomorphism

$$
p_{T}^{-1} \mathcal{T}\left(L_{\xi}\right) \cong \widetilde{W_{T}} \times \widetilde{W_{T}(L)} L / T .
$$

Now apply twisting of coverings as introduced in $\S 3.3$, which leads to the identification Indeed, the choice of $B_{T}$ identifies $p: \mathcal{P} \rightarrow \mathcal{T}$ as a twist of $p_{T}: G / T \rightarrow \mathcal{T}$ and $\mathcal{P}(L) \rightarrow \mathcal{T}(L)$ as a twist of $L / T \rightarrow \mathcal{T}(L)$. Hence, using the isomorphisms (8.15) we may twist the $\widetilde{W_{T}}$-equivariant isomorphism above into a $\widetilde{W}$-equivariant isomorphism

$$
p^{-1} \mathcal{T}\left(L_{\xi}\right) \cong \widetilde{W} \times \widetilde{W(L)} \mathcal{P}(L)
$$

and the result follows.

For an arbitrary element $\xi \in \mathfrak{G}$ with Jordan decomposition $\xi=\xi_{s}+\xi_{n}$ where $\xi_{s}$ is semisimple and $\xi_{n}$ is nilpotent, write $L=L_{\xi}=Z_{G}\left(\xi_{s}\right)^{0}$.

Proposition 8.18. (cf. [Lu4, Lemma 2.4 and its proof]) Let $\xi \in \mathfrak{G}$. We have an isomorphism of $W$-modules,

$$
H^{i}\left(\mathcal{B}_{\xi}^{G}, \mathbb{F}\right) \cong \operatorname{Ind}_{W(L)}^{W} H^{i}\left(\mathcal{B}_{\xi_{n}}^{L_{\xi}}, \mathbb{F}\right),
$$

where $\mathcal{B}^{G}$ denotes the flag variety of $\mathfrak{G}, \mathcal{B}_{\xi}^{G}=\left\{B \in \mathcal{B}^{G} \mid \xi \in \operatorname{Lie}(B)\right\}$, etc.

If $g \in \mathfrak{G}(\mathbb{R})$, then we have an isomorphism of $\widetilde{W}$-modules,

$$
H^{i}\left(\mathcal{B}_{\xi}^{G}, \mathbb{F}\right) \cong \operatorname{Ind} \frac{\widetilde{W}}{W(L)} H^{i}\left(\mathcal{B}_{\xi_{n}}^{L_{\xi}}, \mathbb{F}\right),
$$


where $L_{\xi}=Z_{G}\left(\xi_{s}\right)^{0}$.

Proof. Since $\xi_{s}$ is in the centre of $L, \mathcal{B}_{\xi_{n}}^{L_{\xi}}=\mathcal{B}_{\xi}^{L_{\xi}}$. Hence the first assertion is precisely Lemma 2.4 in [Lu4], the proof of which we now briefly recall. The variety $\mathcal{B}_{\xi}^{G}$ is a disjoint union of copies of $\mathcal{B}_{\xi}^{L}$ indexed by the set of parabolics $P \subset G$ that contain $L$ as a Levi subgroup. In fact, if $U \subset \mathfrak{L}$ is the variety of elements $\zeta \in \mathfrak{L}$ such that $Z_{G}(\zeta) \subset L$, then $U$ is Zariski-dense in $\mathfrak{L}, \xi \in U$ and $\mathcal{B}_{U}^{G}:=\pi^{-1}(U) \subset \widetilde{\mathfrak{G}}$ is a disjoint union of copies of $\mathcal{B}_{U}^{L}$ (defined similarly, with $G$ replaced by $L$ ) which are indexed by the set $\{P \subset G \mid L \subset P\}$ as above. As pointed out in the proof of (8.16), the set $\{P \subset G \mid L \subset P\}$ has a natural transitive $W$-action and is isomorphic to $W / W(L)$.

Now from the intersection cohomology construction of the $W$-action on the cohomology of $\mathcal{B}_{\xi}^{L}$, and of the $W(L)$-action on the cohomology of $\mathcal{B}_{\xi}^{L}$ (see (8.8) et seq.), we see that the first assertion follows from the fact that if $\mathfrak{L}_{r s}$ is the variety of regular semisimple elements of $\mathfrak{L}$, then $\mathfrak{L}_{r s} \subseteq U$, and $\pi_{0}^{-1} \mathfrak{L}_{r s} \subset \widetilde{\mathfrak{G}}$ is $W$-equivariantly isomorphic to $W \times{ }^{W(L)} \widetilde{\mathfrak{L}}_{r s}$ (here $\widetilde{\mathfrak{L}}_{r s}$ is a subvariety of $\widetilde{\mathfrak{L}} \subseteq \widetilde{\mathfrak{G}}$ ).

When $\xi \in \mathfrak{G}(\mathbb{R})$, the same argument shows that we have a $\widetilde{W}$-equivariant isomorphism $\pi_{0}^{-1} \mathfrak{L}_{r s} \cong \widetilde{W} \times \widetilde{W}(L) \widetilde{\mathfrak{L}}_{r s}$, which follows from the isomorphism (8.17) and diagram (8.6). This implies our assertion through application of the intersection cohomology functor, as above.

The next result says that under the Springer correspondence, the nilpotent orbit corresponding to the sign representation is the orbit of $0 \in \mathfrak{G}$. A consequence is the following special case of a result of Borho-MacPherson (which may be found in [LS]).

Lemma 8.21. If $\xi \in \mathfrak{G}$ is nilpotent, the sign representation of $W$ occurs in $H^{i}\left(\mathcal{B}_{\xi}, \mathbb{C}\right)$ if and only if $\xi=0$ and $i=2 N$ (recall $N=\operatorname{dim} \mathcal{B}$, the number of positive roots).

Corollary 8.22. If $\xi \in \mathfrak{G}$, the sign representation of $W$ occurs in $H^{j}\left(\mathcal{B}_{\xi}, \mathbb{F}\right)$ if and only if $\xi$ is semisimple.

Proof of Corollary. Let notation be as above. The multiplicity of the sign representation $\epsilon_{W}$ in $H^{i}\left(\mathcal{B}_{\xi}\right)$ is given by

$$
\begin{array}{rlrl}
\left\langle\epsilon_{W}, H^{i}\left(\mathcal{B}_{\xi}\right)\right\rangle_{W}= & \left\langle\epsilon_{W}, \operatorname{Ind}_{W(L)}^{W} H^{i}\left(\mathcal{B}_{\xi_{n}}^{L}\right)\right\rangle_{W} & & \text { by }(8.18) \\
= & \left\langle\epsilon_{W(L)}, H^{i}\left(\mathcal{B}_{\xi_{n}}^{L}\right\rangle_{W(L)}\right. & & \text { by Frobenius reciprocity } \\
\neq & 0 \text { if and only if } \xi_{n}=0 & \\
& \quad \text { (i.e. } \xi \text { is semisimple) } & \text { by }(8.21) .
\end{array}
$$

Corollary 8.24. Let $\epsilon$ be the sign representation of $W$. For each $i$, we have

$$
\operatorname{Trace}\left(\sigma, H^{i}(\mathcal{B}, \mathbb{F})^{\epsilon}\right)=\operatorname{Trace}\left(\sigma, H_{c}^{i}(\mathcal{P}, \mathbb{F})^{\epsilon}\right),
$$

Both sides are equal to $\operatorname{Trace}\left(\sigma, H_{c}^{i}\left(\mathcal{T}, \mathcal{S}_{\epsilon}\right)\right)$.

Proof. It follows from Lemma 8.13 and Corollary 8.22 that both sides of the equation are zero unless $i=2 N$. In this case we need only check that $\sigma$ acts with the same 
sign, and this follows from Lemma 8.13. The last assertion is immediate from the definition of the coefficient system $\mathcal{S}_{\epsilon}$.

We are now able to give the

Proof of Theorem 8.11. From (7.16), to prove the theorem it suffices to show that

(i) For every $\xi \in \mathfrak{G}$, the stalks of $K^{\bullet}$ and $N^{\bullet}$ have equal Euler-Poincaré characteristics: $\sum_{i \in \mathbb{Z}}(-1)^{i} \operatorname{dim} \mathcal{H}^{i}\left(K^{\bullet}\right)_{\xi}=\sum_{i \in \mathbb{Z}}(-1)^{i} \operatorname{dim} \mathcal{H}^{i}\left(N^{\bullet}\right)_{\xi}$.

(ii) For every $\xi \in \mathfrak{G}(\mathbb{R})=\mathfrak{G}^{\sigma}$, the stalks of $K^{\bullet}$ and $N^{\bullet}$ give equal virtual representations $\sum_{i \in \mathbb{Z}}(-1)^{i}\left[\mathcal{H}^{i}\left(K^{\bullet}\right)_{\xi}\right]=\sum_{i \in \mathbb{Z}}(-1)^{i}\left[\mathcal{H}^{i}\left(N^{\bullet}\right)_{\xi}\right]$.

Let $L=Z_{G}(\xi)^{0}$ and let $T \subset L$ be a maximal torus. As we saw in the proof of Lemma 8.4, the stalk $\mathcal{H}^{i}\left(K^{\bullet}\right)_{\xi}$ is canonically isomorphic to $H_{c}^{i}\left(\mathcal{T}^{\xi}, \mathcal{S}_{\epsilon}\right)$, and if $\xi \in \mathfrak{G}(\mathbb{R})$, then this isomorphism respects the $\Gamma$-action. By $(5.1)$, if $\xi \in \mathfrak{G}(\mathbb{R})$ is semisimple, $\mathcal{T}^{\xi}=\mathcal{T}(L)$ where $\mathcal{T}(L)$ denotes the variety of maximal tori of $L$. Hence for any element $\xi \in \mathfrak{G}$, we have a vector space isomorphism

$$
\mathcal{H}^{i}\left(K^{\bullet}\right)_{\xi} \stackrel{\cong}{\longrightarrow} H_{c}^{i}\left(\mathcal{T}(L), \mathcal{S}_{\epsilon}\right),
$$

and if $\xi \in \mathfrak{G}^{\sigma}$, this is an isomorphism of $\Gamma$-modules.

On the other hand, if we write $\mathbb{F}_{\epsilon}$ for the sign representation of $W$, we have by (8.10), $M_{\epsilon}^{\bullet} \cong R \mathcal{H}^{\circ o m}{ }_{W}\left(\mathbb{F}_{\epsilon}, M^{\bullet}\right)$, where $M^{\bullet}$ is as in Definition 8.9. So for any $\xi \in \mathfrak{G}$, we have, using the notation of (8.18), the following linear isomorphisms, which are isomorphisms of $\Gamma$-modules if $\xi \in \mathfrak{G}(\mathbb{R})$.

$$
\begin{array}{rlrl}
\mathcal{H}^{i}\left(M_{\epsilon}^{\bullet}\right)_{\xi} & \cong \operatorname{Hom}_{W}\left(\mathbb{F}_{\epsilon}, \mathcal{H}^{i}\left(M^{\bullet}\right)_{\xi}\right) & \\
& \cong \operatorname{Hom}_{W}\left(\mathbb{F}_{\epsilon}, H^{i}\left(\mathcal{B}_{\xi}^{G}, \mathbb{F}\right)\right) & \\
& =0 \text { if } \xi \text { is not semisimple, } & & \text { by }(8.22) \\
& \text { and if } \xi \text { is semisimple, } & \\
& \cong \operatorname{Hom}_{W}\left(\mathbb{F}_{\epsilon}, \operatorname{Ind}_{\widetilde{W(L)}}^{\widetilde{W}}\left(H^{i}\left(\mathcal{B}^{L}, \mathbb{F}\right)\right)\right. & & \text { by }(8.18) \\
& \cong \operatorname{Hom}_{W}\left(\mathbb{F}_{\epsilon}, \operatorname{Ind}_{W}^{\widetilde{W}}\left(H_{c}^{4 N(\xi)-i}(\mathcal{P}(L), \mathbb{F})^{*}\right)\right. & & \text { by }(8.13) \\
& \cong \operatorname{Hom}_{W}\left(\mathbb{F}_{\epsilon}, H_{c}^{4 N(\xi)-i}\left(p^{-1} \mathcal{T}(L), \mathbb{F}\right)^{*}\right) & & \text { by }(8.16) \\
& \cong \operatorname{Hom}_{W}\left(\mathbb{F}_{\epsilon}, H_{c}^{4 N(\xi)-i}\left(p^{-1} \mathcal{T}(L), \mathbb{F}\right)\right) & & \text { since } \mathbb{F}_{\epsilon} \text { is self-dual } \\
& \cong H_{c}^{4 N(\xi)-i}\left(\mathcal{T}(L), \mathcal{S}_{\epsilon}\right) &
\end{array}
$$

where $N(\xi)=\operatorname{dim} \mathcal{B}^{L}$.

Hence combining (8.25) and (8.26), we see that for any $\xi \in \mathfrak{G}$, we have linear isomorphisms

$$
\mathcal{H}^{i}\left(M_{\epsilon}^{\bullet}\right)_{\xi} \stackrel{\cong}{\longrightarrow} \mathcal{H}^{4 N(\xi)-i}\left(K^{\bullet}\right)_{\xi}
$$

and that for $\xi \in \mathfrak{G}(\mathbb{R})$, these isomorphisms respect the action of $\Gamma$. This implies a fortiori the statements (i) and (ii) above, and hence completes the proof of Theorem 8.11 . 
Corollary 8.27. The complexes $K^{\bullet}$ and $M_{\epsilon}^{\bullet}=N^{\bullet} \in \mathcal{D}_{c c}^{b}(\mathfrak{G} ; \sigma, \mathbb{F})$ (see Theorem 8.11) have equal characteristic functions $: \mathfrak{G}(\mathbb{R}) \longrightarrow \mathbb{F}$.

This is immediate from Theorem 8.11 and the definition (7.13).

Corollary 8.28. The complexes $\mathcal{F}_{\mathfrak{G}} K^{\bullet}$ and $\mathcal{F}_{\mathfrak{G}} M_{\epsilon}^{\bullet}=\mathcal{F}_{\mathfrak{G}} N^{\bullet} \in \mathcal{D}_{c c}^{b}(\mathfrak{G} ; \sigma, \mathbb{F})$ (see Theorem 8.11) have equal characteristic functions $: \mathfrak{G}(\mathbb{R}) \longrightarrow \mathbb{F}$.

The statement (8.28) follows immediately from Theorem 8.11 and Corollary 7.17.

\section{Proof of the MAIN THEOREM}

Notation is as established in $\S 8$ above. Observe that $V$ is a vector bundle over $\mathcal{T}$ with fibre $\mathfrak{t} \cong \mathbb{A}^{r}$. Moreover since the restriction of the invariant form to $t$ is non-degenerate, $V$ may naturally be identified with its dual bundle $\check{V}$. The inclusion $i: V \hookrightarrow \mathfrak{G} \times \mathcal{T}$ is a map of vector bundles over $\mathcal{T}$, and we may therefore use property (7.7) to compare

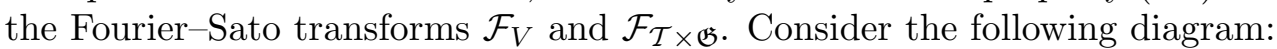

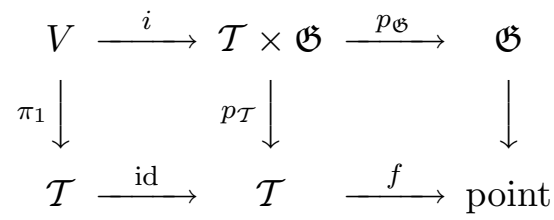

In the notation of Lemma 8.4 and Theorem 8.11, $\rho=p_{\mathfrak{G}} \circ i$. We start by proving

Lemma 9.2. Let $A^{\bullet} \in \mathcal{D}_{c c}^{b}(V ; \sigma, \mathbb{F})$. Then

$$
\mathcal{F}_{\mathfrak{G}}\left(\rho_{!} A^{\bullet}\right)=p_{\mathfrak{G} !} i^{*}\left(\mathcal{F}_{V} A^{\bullet}\right) .
$$

Here $\mathcal{F}_{\mathfrak{G}}$ denotes the Fourier-Sato transform on $\mathcal{D}_{c c}^{b}(\mathfrak{G} ; \sigma, \mathbb{F})$, where $\mathfrak{G}$ is regarded as a self dual vector bundle over a point, and $\mathcal{F}_{V}$ is the Fourier-Sato transform on $\mathcal{D}_{c c}^{b}(V ; \sigma, \mathbb{F})$, where $V$ is regarded as a (self dual) vector bundle over $\mathcal{T}$.

Proof. Since, in (9.1) $i$ is a morphism defined over $\mathbb{R}$ of vector bundles over $\mathcal{T}$, we may apply (7.7) to deduce that for $A^{\bullet} \in \mathcal{D}_{c c}^{b}(V ; \sigma, \mathbb{F})$,

$$
\mathcal{F}_{\mathcal{T} \times \mathfrak{G}}\left(i_{!} A^{\bullet}\right)=\check{i}^{*}\left(\mathcal{F}_{V}\left(A^{\bullet}\right) .\right.
$$

Take $f$ to be the map $f: \mathcal{T} \longrightarrow$ point as shown in the diagram. Then applying (7.10), we obtain for any $C^{\bullet} \in \mathcal{D}_{c c}^{b}(\mathcal{T} \times \mathfrak{G} ; \sigma, \mathbb{F})$

$$
\mathcal{F}_{\mathfrak{G}}\left(p_{\mathfrak{G} !} C^{\bullet}\right)=p_{\mathfrak{G} !}\left(\mathcal{F}_{\mathcal{T} \times \mathfrak{G}}\left(C^{\bullet}\right)\right) .
$$

Using the fact that $\rho=p_{\mathfrak{G}} \circ i$, we may combine (9.4) and (9.5) as follows. For $A^{\bullet} \in \mathcal{D}_{c c}^{b}(V ; \sigma, \mathbb{F})$,

$$
\begin{array}{rlrl}
\mathcal{F}_{\mathfrak{G}}\left(\rho_{!} A^{\bullet}\right) & =\mathcal{F}_{\mathfrak{G}}\left(p_{\mathfrak{G} !}\left(i_{!} A^{\bullet}\right)\right) & \\
& =p_{\mathfrak{G} !} \mathcal{F}_{\mathcal{T} \times \mathfrak{G}}\left(i_{!} A^{\bullet}\right) & & \text { by }(9.5) \\
& =p_{\mathfrak{G} !} i^{*} \mathcal{F}_{V}\left(A^{\bullet}\right) & & \text { by }(9.4),
\end{array}
$$

which is the required statement. 
Next we investigate the Fourier-Sato transform of the complex $K^{\bullet}$. Observe, that the map $\check{i}: \mathcal{T} \times \mathfrak{G} \rightarrow \check{V}$ is given by the restriction of the Killing form

$$
(\mathcal{T} \times \mathfrak{G}) \times_{\mathcal{T}}(\mathcal{T} \times \mathfrak{G})=\mathcal{T} \times(\mathfrak{G} \times \mathfrak{G}) \rightarrow \mathcal{T} \times \mathbb{C}
$$

to the pairing

$$
(\mathcal{T} \times \mathfrak{G}) \times_{\mathcal{T}} V \rightarrow \mathcal{T} \times \mathbb{C} .
$$

Thus $\check{i}(T, \xi)=\left(T, p_{T}(\xi)\right)$, (for $\left.(T, \xi) \in \mathcal{T} \times \mathfrak{G}\right)$, where $p_{T}: \mathfrak{G} \longrightarrow \mathfrak{t}=\operatorname{Lie} T$ is the orthogonal projection with respect to the Killing form. In particular, $\check{i}(T, \xi)=(T, 0)$ if and only if $\xi$ is orthogonal to every $t \in \operatorname{Lie}(T)$.

Proposition 9.7. Let $\mathcal{S}_{\epsilon}^{V}$ be the $\Gamma$-equivariant local system on $V$ introduced in $\S 8$. Then

$$
\mathcal{H}^{i}\left(\mathcal{F}_{\mathfrak{G} \rho !} \mathcal{S}_{\epsilon}^{V}\right)_{\xi} \cong_{\Gamma} H_{c}^{i-2 r}\left(\mathcal{T}_{\xi}, \mathcal{S}_{\epsilon}\right) \otimes \mathbb{F}(r),
$$

where $r=\operatorname{dim} T_{0}$ is the rank of $G$ and $\mathbb{F}(r)$ is the $r^{\text {th }}$ Tate twist of $\mathbb{F}$.

Proof. First observe that by applying (7.8) to the local system $\mathcal{S}_{\epsilon}^{V}=\pi_{1}^{*}\left(\mathcal{S}_{\epsilon}\right)$, we obtain

$$
\mathcal{F}_{V}\left(\mathcal{S}_{\epsilon}^{V}\right) \cong \mathfrak{s h}^{-2 r} \mathfrak{t t}^{-r} \mathcal{S}_{\epsilon}^{0}
$$

where $\mathcal{S}_{\epsilon}^{0}=i_{0 !} \mathcal{S}_{\epsilon}$ is the restriction of $\mathcal{S}_{\epsilon}^{V}$ to $\mathcal{T} \times\{0\} \subseteq V$, extended by zero.

Note that the stalks of $\check{i}^{*}\left(\mathcal{S}_{\epsilon}^{0}\right)$ are given by

$$
\begin{aligned}
\check{i}^{*}\left(\mathcal{S}_{\epsilon}^{0}\right)_{(T, \xi)} & =\left(\mathcal{S}_{\epsilon}^{0}\right)_{\check{i}(T, \xi)} \\
& = \begin{cases}0 & \text { if } \check{i}(T, \xi) \neq(T, 0) \\
\left(\mathcal{S}_{\epsilon}\right)_{T} & \text { otherwise }\end{cases}
\end{aligned}
$$

Hence $\check{i}^{*}\left(\mathcal{S}_{\epsilon}^{0}\right)_{(T, \xi)}$ is zero unless $T \in \mathcal{T}_{\xi}$, and on $\mathcal{T}_{\xi}$ it is $\left(\mathcal{S}_{\epsilon}\right)_{T}$. It follows that

$$
\begin{array}{rlr}
\mathcal{H}^{i}\left(\mathcal{F}_{\mathfrak{G} \rho ! \mathcal{S}_{\epsilon}}^{V}\right)_{\xi} & =\mathcal{H}^{i}\left(p_{\mathfrak{G} ! i^{*}}\left(\mathcal{F}_{V} \mathcal{S}_{\epsilon}^{V}\right)\right)_{\xi} & \\
& \cong H_{c}^{i}\left(p_{\mathfrak{G}}^{-1}(\xi), \check{i}^{*}\left(\mathcal{F}_{V} \mathcal{S}_{\epsilon}^{V}\right)\right) & \\
& \cong H_{c}^{i}\left(p_{\mathfrak{G}}^{-1}(\xi), \mathfrak{s h}^{-2 r} \mathfrak{t} \mathfrak{t}^{-r} \check{i}^{*}\left(\mathcal{S}_{\epsilon}^{0}\right)\right) & \\
& \cong H_{c}^{i-2 r}\left(p_{\mathfrak{G}}^{-1}(\xi), \check{i}^{*}\left(\mathcal{S}_{\epsilon}^{0}\right)\right) \otimes \mathbb{F}(-r) & \\
& \cong H_{c}^{i-2 r}\left(\mathcal{T}_{\xi}, \mathcal{S}_{\epsilon}\right) \otimes \mathbb{F}(-r) & \text { by }(9.9)
\end{array}
$$

The next result follows immediately from (9.7).

Corollary 9.12. With notation as in (9.7) above and Theorem 8.11, if $\xi \in \mathfrak{G}(\mathbb{R})$, we have $\Lambda_{\mathcal{F}_{\mathfrak{G}} K^{\bullet}}(\xi)=(-1)^{r} \Lambda\left(\sigma, \mathcal{T}_{\xi}, \mathcal{S}_{\epsilon}\right)$, where $\Lambda_{\mathcal{F}_{\mathfrak{G}} K} \cdot$ is the characteristic function (see (7.13)) of the Fourier-Sato transform $\mathcal{F}_{\mathfrak{G}} K^{\bullet}$.

We now turn to the Fourier-Sato transform of the object $N^{\bullet} \in \mathcal{D}_{c c}^{b}(\mathfrak{G} ; \sigma, \mathbb{F})$.

Proposition 9.13. For any representation $E$ of $W$, let $M_{E}^{\bullet}$ be the complex of (8.10). Then in the notation of (8.11), we have

$$
\mathcal{F}_{\mathfrak{G}}\left(M_{\epsilon}^{\bullet}\right)=\left.\mathfrak{t t}^{N-\operatorname{dim} \mathfrak{G}}\left(M_{1}^{\bullet}[-\operatorname{dim} \mathfrak{G}-r]\right)\right|_{\mathfrak{G}_{\text {nil }}},
$$


where $r=\operatorname{dim} T_{0}=\operatorname{rank}(G)$ and $\left.\right|_{\mathfrak{G}_{\text {nil }}}$ denotes the restriction to the nilpotent cone, extended by zero and $N=\operatorname{dim} \mathcal{B}$ is the number of positive roots of $G$.

Proof. It follows from (7.11) and [Sho, Proposition 17.7] (the right side of which should have $\left(\nu_{G}\right)$ added $)$ that since $M_{\epsilon}^{\bullet}[\operatorname{dim} \mathfrak{G}]$ is perverse,

$$
\left.(\mathfrak{s h})^{\operatorname{dim} \mathfrak{G}} \mathcal{F}_{\mathfrak{G}}\right)^{-1}\left(M_{\epsilon}^{\bullet}[\operatorname{dim} \mathfrak{G}]\right)=\left.\mathfrak{t t}^{N}\left(M_{1}^{\bullet}[\operatorname{dim} \mathfrak{G}-r]\right)\right|_{\mathfrak{G}_{\text {nil }}} .
$$

But from (7.3), noting that $\mathcal{F}, \mathfrak{s h}$ and $\mathfrak{t t}$ all commute,

$$
\begin{aligned}
\left(\mathfrak{s h}^{\operatorname{dim} \mathfrak{G}} \mathcal{F}_{\mathfrak{G}}\right)^{-1}= & \mathfrak{s h}^{-\operatorname{dim} \mathfrak{G}} \mathcal{F}_{\mathfrak{G}}^{\prime} \\
& =\mathfrak{t t}^{\operatorname{dim} \mathfrak{G}} \circ \mathfrak{s h} \mathfrak{d i m ~ G}_{\operatorname{dinv}}{ }^{*} \circ \mathcal{F}_{\mathfrak{G}} .
\end{aligned}
$$

Combining (9.14) with (9.15), and using the fact that inv* leaves $M_{1}^{\bullet}$ invariant, we obtain the statement of the proposition.

We are now able to complete the

Proof of Theorem 1.10. It follows from (8.28) that the complexes $\mathcal{F}_{\mathfrak{G}} K^{\bullet}$ and $\mathcal{F}_{\mathfrak{G}} M_{\epsilon}^{\bullet}$ have the same characteristic functions, i.e. that, in the notation of $(7.13), \Lambda_{\mathcal{F}_{\mathfrak{G}}\left(K^{\bullet}\right)}=$ $\Lambda_{\mathcal{F}_{\mathfrak{G}}\left(M_{\epsilon}\right)}$. But by $(9.12), \Lambda_{\mathcal{F}_{\mathfrak{G}} K} \bullet(\xi)=(-1)^{r} \Lambda_{c}\left(\sigma, \mathcal{I}_{\xi}, \mathcal{S}_{\epsilon}\right)$, while from (9.13) it follows that $\mathcal{F}_{\mathfrak{G}}\left(M_{\epsilon}^{\bullet}\right)$ is the constant sheaf $\mathbb{F}$ on $\mathfrak{G}_{\text {nil }}$ extended by zero, concentrated in degree $-(\operatorname{dim} \mathfrak{G}+r)$, with $\sigma$ acting as $(-1)^{\operatorname{dim} \mathfrak{G}+N}$. It follows that for $\xi \in \mathfrak{G}(\mathbb{R})$,

$$
\Lambda_{c}\left(\sigma, \mathcal{T}_{\xi}, \mathcal{S}_{\epsilon}\right)= \begin{cases}(-1)^{N} & \text { if } \xi \in \mathfrak{G}_{\text {nil }} \\ 0 & \text { otherwise }\end{cases}
$$

as required. Taking (5.2) into account, the statement about weighted Euler characteristics follows from Theorem 4.5.

\section{Explicit COMPUTATIONS FOR $\mathrm{SL}_{2}$, AND CONCLUding REMARKS}

10.1. The case of $\mathbf{S L}_{2}$. We carry out explicit computations to illustrate Theorem 1.10 in the case $G=\mathrm{SL}_{2}$.

Take $G=\mathrm{SL}_{2}(\mathbb{C})$ with $\sigma$ acting as coordinate-wise complex conjugation and let $T_{0}$ be the diagonal subgroup. In this case $G$ is split, so in particular, $\mathcal{P} \cong G / T_{0}$ over $\mathbb{R}$ and we may indentify $W$ with $W_{0}=N_{G}\left(T_{0}\right) / T_{0}$.

We have $W_{0}=\{1, r\}$, represented by

$$
\left\{1=\left(\begin{array}{ll}
1 & 0 \\
0 & 1
\end{array}\right), r=\left(\begin{array}{cc}
0 & 1 \\
-1 & 0
\end{array}\right)\right\} \subset N_{G}\left(T_{0}\right) \text {. }
$$

Let

$$
\xi_{0}:=\left(\begin{array}{cc}
1 & 0 \\
0 & -1
\end{array}\right) \in \mathfrak{G}
$$

Then the map $G \rightarrow \mathfrak{G}$ given by $g \mapsto g \xi_{0} g^{-1}$ induces an isomorphism of $\mathcal{P}$ with the $G$-orbit of $\xi_{0}$. Since $\xi_{0}$ is semisimple, this $G$-orbit consists of all $2 \times 2$ matrices with the same characteristic polynomial as $\xi_{0}$. Hence we get an embedding

$$
\mathcal{P}=\left\{\left(\begin{array}{ll}
a & b \\
c & -a
\end{array}\right) \mid a^{2}+b c=1\right\} \subset \mathfrak{G} \cong \mathbb{A}^{3} .
$$


The $W_{0}$-action on $\mathcal{P}$ is given by the involution

$$
g \xi_{0} g^{-1} \mapsto g r \xi_{0} r^{-1} g^{-1}
$$

Hence if we write the matrix in (10.1) as $(a, b, c) \in \mathbb{A}^{3}$, the non-trivial element $r$ of $W_{0}$ takes $(a, b, c)$ to $(-a,-b,-c)$. It follows that $\mathcal{T} \cong\left\{[a, b, c] \in \mathbb{P}^{2} \mid a^{2}+b c \neq 0\right\}$ and $p: \mathcal{P} \longrightarrow \mathcal{T}$ is given by $(a, b, c) \mapsto[a, b, c]$.

For $\xi \in \mathfrak{G}$, define $\mathcal{P}^{\xi}:=p^{-1} \mathcal{T}^{\xi}$ and $\mathcal{P}_{\xi}:=p^{-1} \mathcal{T}_{\xi}$.

The Killing form on $\mathfrak{G}$ is given by $\langle\xi, \eta\rangle=\operatorname{Trace}(\xi \eta)$, and hence for $\xi=\left(\begin{array}{cc}x & y \\ z & -x\end{array}\right) \in$ $\mathfrak{G}$ the subvariety $\mathcal{P}_{\xi} \subset \mathcal{P}$ is given by

$$
\mathcal{P}_{\xi}:=\left\{\left(\begin{array}{ll}
a & b \\
c & -a
\end{array}\right) \in \mathcal{P} \mid 2 x a+y c+z b=0\right\} .
$$

Suppose now that $\xi \in \mathfrak{G}(\mathbb{R})$. Since $\mathcal{T}_{\xi}=\mathcal{P}_{\xi} / W_{0}$, and $W_{0}=\{1, r\}$, the space $\mathcal{T}_{\xi}(\mathbb{R})$ of real points admits a decomposition

$$
\mathcal{T}_{\xi}^{\Gamma}=p\left(\mathcal{P}_{\xi}^{\sigma}\right) \cup p\left(\mathcal{P}_{\xi}^{r \sigma}\right)
$$

Clearly $\mathcal{P}_{\xi}^{\sigma}=\mathcal{P}_{\xi}(\mathbb{R})$, the space of real points of $\mathcal{P}_{\xi}$, while

$$
\mathcal{P}_{\xi}^{r \sigma}=\left\{\left(\begin{array}{ll}
a & b \\
c & -a
\end{array}\right) \in \mathcal{P}_{\xi} \mid a, b, c \in \sqrt{-1} \mathbb{R}\right\}
$$

It is easy to check that $\mathcal{T}_{\xi}^{+}:=p\left(\mathcal{P}_{\xi}^{\sigma}\right)$ is the space of tori in $\mathcal{T}_{\xi}(\mathbb{R})$ of type +1 , while $p\left(\mathcal{P}_{\xi}^{r \sigma}\right):=\mathcal{T}_{\xi}^{-}$is the space of tori in $\mathcal{T}_{\xi}(\mathbb{R})$ of type -1 .

Now Theorem 1.10 asserts that

$$
\Lambda_{\mathrm{c}}\left(\sigma, \mathcal{T}_{\xi}, \mathcal{S}_{\epsilon}\right)=\sum_{\substack{C \subset \mathcal{T}_{\xi}^{\Gamma} \\ \text { connected } \\ \text { component }}} \chi(C) \cdot \varepsilon(C)=\chi\left(\mathcal{T}_{\xi}^{+}\right)-\chi\left(\mathcal{T}_{\xi}^{-}\right),
$$

is -1 if $\xi$ is nilpotent, and 0 otherwise. On the other hand, since $\mathcal{P}_{\xi}^{\sigma} \rightarrow \mathcal{T}_{\xi}^{+}$and $\mathcal{P}_{\xi}^{r \sigma} \rightarrow \mathcal{T}_{\xi}^{-}$are unramified double coverings, we have

$$
\Lambda_{\mathrm{c}}\left(\sigma, \mathcal{T}_{\xi}, \mathcal{S}_{\epsilon}\right)=\frac{1}{2}\left(\chi\left(\mathcal{P}_{\xi}^{\sigma}\right)-\chi\left(\mathcal{P}_{\xi}^{r \sigma}\right)\right)
$$

In the following table we list all cases, verifying the Theorem. For $\xi \in \mathfrak{G}(\mathbb{R})$, we represent $\mathcal{P}_{\xi}^{\sigma}$ by the equations defining it as subset of $\mathfrak{G}(\mathbb{R}) \cong \mathbb{R}^{3}$ and we represent 
$\mathcal{P}_{\xi}^{r \sigma}$ by the equations defining it as subset of $\sqrt{-1} \mathbb{R}^{3}$.

\begin{tabular}{|c|c|c|c|c|c|}
\hline$\xi$ & $\| \Lambda_{\mathrm{c}}\left(\sigma, \mathcal{T}_{\xi}, \mathcal{S}_{\epsilon}\right)$ & $\mathcal{P}_{\xi}^{\sigma}$ & $\chi\left(\mathcal{P}_{\xi}^{\sigma}\right)$ & $\mathcal{P}_{\xi}^{w \sigma}$ & $\chi\left(\mathcal{P}_{\xi}^{w \sigma}\right)$ \\
\hline$\left(\begin{array}{ll}0 & 0 \\
0 & 0\end{array}\right)$ & -1 & $a^{2}+b c=1$ & 0 & $a^{2}+b c=-1$ & 2 \\
\hline$\left(\begin{array}{ll}0 & 1 \\
0 & 0\end{array}\right)$ & -1 & $\left\{\begin{array}{l}a^{2}=1 \\
c=0\end{array}\right.$ & -2 & $\emptyset:\left\{\begin{array}{l}a^{2}=-1 \\
c=0\end{array}\right.$ & 0 \\
\hline$\left(\begin{array}{cc}1 & 0 \\
0 & -1\end{array}\right)$ & 0 & $\left\{\begin{array}{l}a=0 \\
b c=1\end{array}\right.$ & -2 & $\left\{\begin{array}{l}a=0 \\
b c=-1\end{array}\right.$ & -2 \\
\hline$\left(\begin{array}{cc}0 & 1 \\
-1 & 0\end{array}\right)$ & 0 & $\begin{array}{l}b=c \\
a^{2}+b^{2}=1\end{array}$ & 0 & $\left\{\begin{array}{l}b=c \\
a^{2}+b^{2}=-1\end{array}\right.$ & 0 \\
\hline
\end{tabular}

10.2. Concluding remarks and open problems. The results presented here raise several questions, among which are the following.

The formula for $\Lambda_{c}\left(\sigma, \mathcal{T}^{\xi}, \mathcal{S}_{\epsilon}\right)$ bears a striking resemblance to the character formula for the Steinberg representation of a reductive group over $\mathbb{F}_{q}$, with ' $q$ repaced by -1 '. We therefore ask

10.2. Is there a representation of $G(\mathbb{R})$ with a "trace" whose value at $x \in G(\mathbb{R})$ is $\pm \Lambda_{c}\left(\sigma, \mathcal{T}^{x}, \mathcal{S}_{\epsilon}\right)$ ?

In general, there are other $\sigma$-equivariant local systems on $\mathcal{T}$ and on $\mathcal{B}$. For example when $G=\mathrm{SL}_{n}(\mathbb{C})$ and $\sigma$ is complex conjugation, all the local systems $\mathcal{S}_{\rho}$ are $\sigma$ equivariant. The following question therefore arises naturally.

10.3. Compute $\Lambda_{c}\left(\sigma, \mathcal{T}^{\xi}, \mathcal{S}_{\rho}\right)$ for other representations $\rho$ of $W$, There are analogies with the case of $\mathbb{F}_{q}$ which suggest that the values of Green functions at $q=-1$ may be involved.

In the case of a finite field, the analogues of the results here were proved by using characteristic functions, without reference to sheaves. This was possible because there is a notion of "Fourier transform" for functions on a vector space over $\mathbb{F}_{q}$, whose properties suffice for the proof. We therefore ask

10.4. Is there a "Fourier transform" on the space of constructible functions on a real vector bundle $E$, which is defined without reference to sheaves, which may be used to prove Theorem 1.10 directly, in analogy with the finite characteristic case (cf. [Le2])? Such a transform on the space of constructible functions would presumably have the property that for $K^{\bullet} \in \mathcal{D}_{c c}^{b}(E ; \sigma, \mathbb{F})$, the Fourier transform of $\chi_{K} \bullet$ is $\chi_{\mathcal{F}_{E}\left(K^{\bullet}\right)}$. 


\section{REFERENCES}

[BBD] A.A. Beilinson, J. Bernstein et P. Deligne, "Faisceaux pervers (French) [Perverse sheaves]",Analysis and topology on singular spaces, I (Luminy, 1981), Astérisque 100, Soc. Math. France, Paris, (1982), 5-171

[Bo] A. Borel, "Linear algebraic groups. Second edition." Graduate Texts in Mathematics, 126, Springer-Verlag, New York, (1991).

[BT] A. Borel and J. Tits, "Groupes réductifs (French)", Inst. Hautes Études Sci. Publ. Math. 27, (1965), 55-150.

[Br] G. Bredon, "Introduction to compact transformation groups", Pure and Appl. Math., 46, Academic Press, 1972.

[SGA3] M. Demazure et A. Grothendieck, "Séminaire de Géométrie Algébrique du Bois Marie 1962/64 (SGA 3)", Lecture Notes in Mathematics, 153, Springer-Verlag, Berlin, 1962/1964.

[Gi] Ph. Gille, "Type des tores maximaux des groupes semi-simples", J. Ramanujan Math. Soc. 19 (2004), 213-230.

[H] H. Hironaka, "Triangulations of algebraic sets", Algebraic geometry (Arcata 1974), Proc. Sympos. Pure Math. 29, Amer. Math. Soc., Providence, R.I., (1975), 165-185.

[Kaw91] K. Kawakubo, "The theory of transformation groups", Oxford University Press, 1991.

[KS] M. Kashiwara and P. Schapira, "Sheaves on manifolds." Grundlehren der Mathematischen Wissenschaften 292. Springer-Verlag, Berlin, 1994

[Le1] G.I. Lehrer, "Rational tori, semisimple orbits and the topology of hyperplane complements", Comment. Math. Helv. 67 (1992), 226-251.

[Le2] G.I. Lehrer, "The space of invariant functions on a finite Lie algebra", Trans. Amer. Math. Soc. 348 (1996), 31-50.

[LS] G.I. Lehrer and T. Shoji, "On flag varieties, hyperplane complements and Springer representations of Weyl groups", J. Austral. Math. Soc. Ser. A 49 (1990),449-485.

[€] S. Łojasiewicz, "Triangulation of semi-analytic sets", Ann. Scuola Norm. Sup. Pisa (3) 18 (1964), 449-474.

[Lu1] G. Lusztig, "Intersection cohomology complexes on a reductive group", Inventiones 75 (1984), 205-272.

[Lu2] G. Lusztig, "Character Sheaves", Advances in Math. 56 (1985), 193-237, 57 (1985), 226-315, 59 (1986), 1-63, 61 (1986), 103-165.

[Lu3] G. Lusztig, "Green Functions and Character Sheaves", Annals of Math. 131 (1990) 355-408.

[Lu4] G. Lusztig, "An induction theorem for Springer's representations", Representation theory of algebraic groups and quantum groups, Adv. Stud. Pure Math. 40, Math. Soc. Japan, Tokyo (2004) 253-259.

[PS] D.H. Park and D.Y. Suh, "Equivariant semi-algebraic triangulations of real algebraic $G$ varieties", Kyushu J. Math. 50, (1996), 179-205.

[R] M.S.Raghunathan, "Tori in quasi-split-groups", J. Ramanujan Math. Soc. 19 (2004), 281-287.

[Se] J.-P. Serre, "Galois cohomology", Springer-Verlag, 1997.

[She] D. Shelstad, "Characters and inner forms of a quasi-split group over $\mathbb{R}$ ", Compositio Math. 39 (1979), 11-45.

[Sho] T. Shoji, "Geometry of orbits and Springer correspondence", Astérisque 168 (1988), 61-140.

[Spr] T.A. Springer, "Linear algebraic groups", Second edition, Progress in Mathematics 9, Birkäuser Boston, Inc., Boston, MA, (1998).

[W] H. Whitney, "Elementary structure of real algebraic varieties", Ann. of Math. (2) 66, (1957), 545-556.

School of Mathematics and Statistics, The University of Sydney, Sydney, Australia 2006.

Department of Mathematics, Kuleuven, Celestijnenlaan 200B, B-3001 Leuven (HevERLEe), Belgium. 